\title{
Resolving Small Objects Using Seismic Traveltime Tomography
}

\author{
David Carl Loveday
}

Thesis submitted to the faculty of the Virginia Polytechnic Institute and State University in partial fulfillment of the requirements for the degree of

Master of Science

in

Geoscience

John A. Hole

J. Arthur Snoke

Martin C. Chapman

August 24, 2007

Blacksburg, VA

Keywords: traveltime picks, tomography, spatial resolution, detection of small objects, seismic refraction, Fresnel zone

(C) David C. Loveday, 2007 


\title{
Resolving Small Objects Using Seismic Traveltime Tomography
}

\author{
David Carl Loveday
}

\begin{abstract}
It is often claimed that the first Fresnel zone associated with the dominant frequency represents the spatial resolution limit of traveltime tomography. We show, however, that the relevant Fresnel limit for tomographic resolution is the maximum, not the dominant frequency in the data. For physically realizable causal wavelets, the maximum frequency is infinite. In practice, noise lowers the effective possible maximum frequency. To demonstrate these points, synthetic seismic data were generated for traveltime picking and inversion for a single, small velocity anomaly embedded in a homogeneous background velocity. A variety of traveltime picking techniques were tested and compared for their ability to detect the presence of objects smaller than that Fresnel zone associated with the dominant frequency. All methods produced accurate ray-theoretical (infinite-frequency) picks from noise-free seismic data for objects smaller than the dominant-frequency Fresnel zone. For the lowest dominant frequencies with Fresnel zones many times larger than the object, picking methods that focus on features along the onset of the first arrival were the most accurate, while cross-correlation with a known wavelet preformed less accurately. First-onset picking methods perform better because they take advantage of the highest frequencies in the data, whereas the correlation wavelet is typically in line with the dominant frequency. All methods successfully detected the presence of objects smaller than a wavelength. The inversion of the traveltime picks from the different picking methods always recovered the position and shape of the object. Random noise at a range of signal-to-noise ratios was then added to
\end{abstract}


the seismic data and the data were repicked. Pick times with different noise realizations are statistically centered on the noise-free pick, not the time that would be recorded in the absence of the object. Trace stacking prior to picking or the averaging of many picks improves the signal-to-noise ratio and can extract signal that is not detected on an individual pick. An averaging of traveltime picks also occurs during tomographic inversion. This inherent signal-to-noise improvement allows tomography to image objects that are undetectable in individual trace picks. The resolution of tomography is limited not by the Fresnel zone associated with the dominant frequency, but by the accuracy of the traveltime picks. Resolution is further improved by dense ray coverage. 


\section{Acknowledgements}

This work would not have been possible without scholarship support from Chevron Corporation, Matthew Mikulich, and the Aubrey-Orange endowment. Matthias Imhof supplied the synthetic data and software as well as much advice. The author would like to thank Ying Zhou for her constructive comments. Jake Beale was a continual source of knowledge and encouragement. The author would also like to thank the technical and administrative staff of Virginia Tech. 


\section{Table of Contents}

Abstract.....................................................................ii

Acknowledgements.....................................................iv

Table of Contents.......................................................v

List of Figures..........................................................vi

Introduction.............................................................1

Background..................................................

First-break Picks with Interfering Casual Wavelets......................5

Motivation...................................................... 8

Synthetic Data.........................................................9

Noise-free Picks for Small Objects....................................12

Noise-free Tomography of Small Objects..............................14

Noise and Single Trace Picking.......................................17

Tomography Inversion of Noisy Data...................................19

Conclusion..................................................................22

References.............................................................24

Figures..................................................................27

Appendix............................................................ 53 


\section{List of Figures}

Figure 1: Raypaths from a source to a receiver for a uniform background velocity.

Figure 2: Berlage wavelet and its spectrum and illustration of picking criteria...... .28

Figure 3: Two equal amplitude wavelets with decreasing frequency and increasing

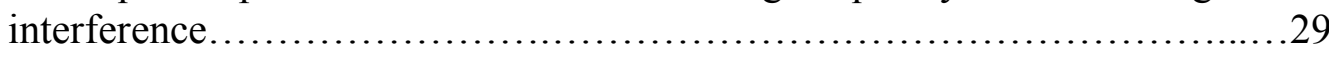

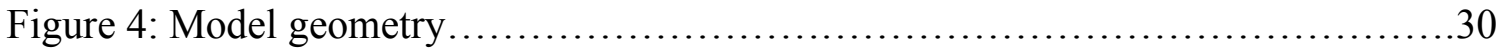

Figure 5: Synthetic Green's functions for source and receivers highlighted in Figure 3 .

Figure 6: Green's functions for 0 degree source-receiver pair for fast, no, and slow object

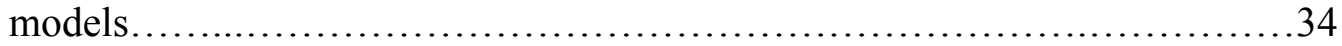

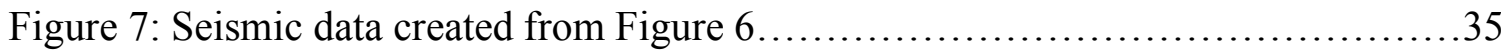

Figure 8: Seismic data at a range of frequencies for the 0 degree source-receiver pair for (a) fast object and (b) slow object..........................................36

Figure 9: Picking results for noise-free data of Figure 8 for a fast object and slow object as a function of frequency....................................................

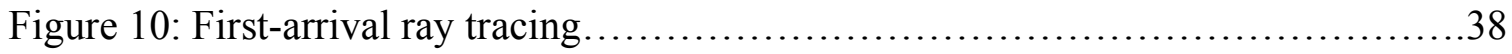

Figure 11: Example tomograms for noise-free data, 360 shots and 360 receivers, and a range of frequencies $39-40$

Figure 12: Width of anomaly resolved by tomography as a function of frequency for fast (blue) and slow (red) objects....

Figure 13: Example of the same trace with different $\mathrm{S} / \mathrm{N}$ added.

Figure 14: Histogram of 50\% threshold picking results for (a) fast and (b) slow object data at different $\mathrm{S} / \mathrm{N}$.

Figure 15: Standard deviation of pick distributions from Figure 14 as a function of $\mathrm{S} / \mathrm{N}$ on logarithmic-logarithmic scale for fast (blue dots) and slow (pink dots) noisy picks

Figure 16: Histogram of 50\% threshold picking results for (a) fast and (b) slow data at different stacking folds. 
Figure 17: Standard deviation of pick distributions from Figure 16 as a function of stacking fold on logarithmic-logarithmic scale for fast (blue dots) and slow (pink dots) stacked picks.............................................46

Figure 18: Histogram of 50\% threshold picking results for (a) fast and (b) slow object data at different averaging folds........................................47

Figure 19: Standard deviation of pick distributions from Figure 18 as a function of averaging fold on logarithmic-logarithmic scale for fast (blue dots) and slow (pink dots) averaged picks............................................48

Figure 20: Example tomograms for noise-free (a) fast and (b) slow object 50\% threshold picks with decreasing ray coverage .......................................49

Figure 21: Example tomograms for (a) fast and (b) slow object noisy data at 360 sources and receivers........................................................ 50

Figure 22: Example tomograms of fast and slow object data at low $\mathrm{S} / \mathrm{N}$ but with excellent ray coverage.

Figure 23: Example tomograms for (a) fast and (b) slow object data at $\mathrm{S} / \mathrm{N}$ of 4 at different ray coverages. 


\section{Introduction}

Traveltime tomography inverts traveltime picks, most often from first-arrivals, to create an image of the internal velocity structure of the earth. The spatial resolution or quality of the obtained image is a function of the number of sources and receivers and their spatial configuration, as well as the accuracy and precision of the picks. Classical traveltime tomography uses raypaths from sources to receivers to model traveltimes. Ray theory assumes infinite-frequency energy traveling along infinitesimally thin rays. One topic of recent interest is the accuracy of traveltime picks for realistic data with limited bandwidth and the impact of this on tomographic resolution of small objects. Estimates of traveltime tomography spatial resolution limits evolved from a few wavelengths (e.g. Williamson, 1991) to a Fresnel zone (e.g. Cerveny et al., 1992; Nolet et al., 2000; Dahlen et al., 2000), and the idea that picking accuracy may be a function of picking technique (Gundmundsson, 1996). Recently, the Fresnel zone has been widely referred to as the spatial limit beyond which the ability to detect or resolve small objects is lost or severely

limited (Marquering et al., 1999; Gundmundsson, 1996; Baig et al., 2003; Snieder et al, 1996). In most cases (see references cited below) the frequency used to define the Fresnel zone is the dominant frequency in the signal. In most applications, the size of the dominant-frequency Fresnel zone is depressingly large: several hundred kilometers for global earthquake data, several kilometers for refraction data in the upper $10 \mathrm{~km}$ of the crust, and tens of meters for cross-borehole data in petroleum applications. This project tests this claim to determine if objects smaller than a Fresnel zone are detectable and whether they may be tomographically recovered. 


\section{Background}

Geometric ray theory is a common tool used to efficiently approximate wave propagation through complex media. This technique models body waves as traveling from source-to-receiver along rays of infinitesimal width. The arrival time depends only on the wave speed along the ray. The theory is only valid for an infinite-frequency wave and does not account for the lower-frequency effects observed in realistic band-limited seismic data. Finite-frequency waves have a width about the ray in which structure may affect the observed ground shaking. The Fresnel zone is defined as the volume surrounding the ray within which scattered energy arrives at the receiver within one-half period of the wave [e.g. Kravstov et al., 1980]. A scatterer (or region of space) is said to lie within the first Fresnel zone if it satisfies the inequality:

$$
\left(t\left(r_{s}, r_{\text {scat }}\right)+t\left(r_{\text {scat }}, r_{g}\right)\right)-t\left(r_{s}, r_{g}\right) \leq T / 2=1 / 2 f
$$

where $t$ is the traveltime, $r_{s}, r_{\text {scat }}, r_{g}$ are the positions of the source, scatterer, and receiver (see Figure 1), $T$ is the period, and $f$ is the frequency. The Fresnel zone is larger for waves of lower frequency. If the scattered energy of a monochromatic wave arrives within less than half a period of the shortest path, constructive wavelet interference will change the characteristics of the first half-cycle of the recorded arrival. Delays between a half and full period will cause destructive interference in the second half-cycle, but will not affect the character of the first half-cycle. Times greater than a period will see no interference of the first full cycle of the wavelet. A region with faster or slower velocity that lies on or near the ray path will advance or delay some of the wave energy. Perturbations to a wave caused by small objects quickly decay in amplitude as the wave propagates 
beyond the object, causing the wave to "heal". At greater distances, the wavefront looks increasingly like the unperturbed wavefront. The rate of decay is a function of the initial size and amplitude of the wave perturbation, so those associated with small objects decay more quickly. Nolet et al. (2000) show that wavefront healing has a significant effect on tomographic resolution for objects smaller than a Fresnel zone.

The Fresnel zone was initially defined as the boundary between constructive and destructive interference of a monochromatic wave. Seismic traveltime picks are not made on monochromatic waves (nor in the frequency domain) but by looking at some portion of a brief time-domain wavelet with non-zero bandwidth. Most of the papers that cite the Fresnel zone as the resolution limit for tomography explicitly or implicitly assume that the relevant frequency is the dominant or central frequency, including both active sources in industry applications [e.g. Williamson, 1991; Pratt, 1999; Thore et al., 1999; Spetzler et al., 2002; Sheng et al., 2003] and earthquake sources for global imaging [e.g. Snieder et al., 1996; Marquering et al., 1999; Hung et al., 2001; Baig et al., 2004].

The half-period criterion of the Fresnel zone refers to plus-minus wavelet interference, which may not be relevant to picking methods that depend upon relative amplitude thresholds or other criteria near the onset of the wavelet. Marquering et al. (1999) point out that traditional hand picks of the first break (first arrival) actually take advantage of frequencies higher than the dominant frequency.

Different branches of seismology have different picking methods popular within their communities. Refraction and cross-borehole seismology most often pick the first 
onset of energy of a causal wavelet. Local and regional earthquake catalogs also usually contain picks of the initial onset of the first arrival. Cross-correlation of waveforms from nearby earthquakes is used to obtain more precise relative traveltimes (e.g. Hansen et al., 2006; Richards et al., 2005). The resulting earthquake locations may be centroids rather than hypocenters. The locations are more precise relative to nearby earthquakes but are not more accurate in an absolute sense. Global earthquake traveltimes are most commonly obtained by cross-correlation with synthetically generated waveforms (e.g. Melleors, 2004; Lin, 2007). Reflection seismology usually picks the peaks of zero-phase wavelets created by deconvolution or by cross-correlation with a known source wavelet. Each picking method is sensitive in different ways to scattered energy.

Baig et al. (2004) showed that where a 3-D heterogeneity is smaller than the Fresnel zone based on the dominant period, there is a difference between crosscorrelation and ray-theoretical traveltimes. Gundmundsson (1996) showed that under certain conditions, first onset picks are accurate to $\mathrm{T} / 4$ (where $\mathrm{T}$ is the dominant period), while cross-correlation techniques can measure traveltimes to an accuracy approaching $\mathrm{T} / 2$. This discrepancy arises from the cross-correlation's reliance on the full time window of the pilot wavelet which may include scattered energy delayed (behind the direct arrival) as long as the full wavelet. Commonly, the pilot wavelet is in line with the dominant frequency in the data. First-onset picking focuses on a much narrower time window near the first break which can only include earlier scattering times. Still, these resolution claims are framed around the dominant frequency of a narrow-band wavelet.

All physically realizable wavelets are casual, and seismic wavelets from explosions usually have very limited duration. Causal wavelets have a true first break 
and extend to infinite frequency (Bracewell, 1995 p.141). Causality means no signal before $t=0$, which requires that the wavelet or, more likely, one of its derivatives includes a step discontinuity. The Fourier transform of a step discontinuity (or its derivatives or integrals) has infinite bandwidth, even if the high frequencies are very small. In practice, the maximum frequency in the data is limited by sampling, the instrument responses, and noise. However, the effective maximum frequency remains much higher than either the central or dominant frequency. The infinite-frequency (ray) time can always be picked in a noise-free universe; it is the time of the first non-zero signal. In a realistic noisy universe, the energy of the signal must be above some noise-defined detection threshold and the pick is affected by scattered energy arriving in the time it takes for the wavelet to exceed that threshold. This time delay, defined by the noise-level and wavelet shape, defines an effective maximum frequency and the relevant Fresnel zone. Noisesuppression techniques such as pick averaging may raise this effective maximum frequency.

\section{First-Break Picks with Interfering Causal Wavelets}

To simulate the picking of first arrivals requires a causal source wavelet. The Berlage wavelet (Figure 2) (Aldridge, 1990) is causal, minimum phase, and differentiable to a high order. The wavelet is described by the equation:

$$
w(t)=A H t^{n} e^{-\alpha t} \sin \left(2 \pi f_{0} t+\Phi_{0}\right)
$$

where $H(t)$ is the Heaviside unit step function $[H(t)=1$ for $t>0]$. The exponential decay factor $\alpha$ and the time exponent $n$ are nonnegative real constants, $\Phi_{0}$ is the initial phase 
angle, and $f_{0}$ is the dominant frequency (Figure $2 \mathrm{~b}$ ). For our simulations, $n$ is set equal to $1, \Phi_{0}$ is set to 0 , and $\alpha$ is set to be $0.1 \%$ greater than the minimum-phase condition (Aldridge, 1990).

Picking was performed automatically using several simple criteria (Figure 2). The simplest is to pick the first recognizable peak of the wavelet; for a minimum-phase wavelet, this is the largest peak. Searching backwards from the first peak, threshold picks were made from $90 \%$ to $10 \%$ of the peak amplitude. Manual first-break picks usually consist of a similar amplitude threshold or the zero-crossing ( $0 \%$ threshold) immediately before the first signal above a larger threshold. An alternative criterion is the maximum-slope (inflection point or maximum derivative) prior to the first peak. A separate picking algorithm uses cross-correlation, where picks were computed by crosscorrelating with the known pilot wavelet. For real data, the pilot wavelet is not known a priori, but is approximately derived from the data or assumptions about the source. In order to compare different criterion, each pick was adjusted for the delay from the initial onset (first non-zero sample) caused by the picking method. This was achieved by picking the pilot Berlage wavelet using the same criterion and subtracting the pilot pick delay from the trace pick. If the trace consists of only a single arrival of a clean wavelet, then all of the adjusted picks will give the same result - the first non-zero sample in the signal. If the trace contains multiple interfering arrivals, differences among picks will be due solely to differences in the shape of the waveform.

To test the effectiveness of these picking criteria on first arrival times, they were applied to traces consisting of two overlapping equal-amplitude wavelets (Figure 3a). These are the convolution of the Berlage wavelet at a range of dominant frequencies $\left(f_{0}\right)$ 
with two identical spike impulses separated by a time $\Delta \mathrm{t}$, which is held constant. As the dominant frequency is lowered, the duration of each wavelet increases to where the first arrival waveform overlaps the onset of the second arrival.

At the highest dominant frequencies, there are two distinct wavelets. As the overlap of the wavelets increases, the first peak shifts later in time, and the waveform visibly broadens. When the overlap of the wavelets is on the order of the Fresnel zone half-period criterion based on the dominant frequency, the data begins to look more like a single multi-lobed peak. At the lowest frequencies, the data appear to be a single wavelet. The true first break, a threshold pick of near zero percent, is of course unaffected by the choice of the dominant frequency. However, for a fixed sample rate, the change in amplitude of the first point after the true arrival time will decrease. As the threshold is increased, the pick becomes more sensitive to the second wavelet (Figure 3b). The second wavelet affects the pick if it starts prior to the uncorrected pilot pick time (the time between the onset of energy and the pick shown in Figure 2). Lower amplitude thresholds are least affected, with pick times closest to the first break. The first peak (or $100 \%$ threshold) is the more affected by interference. The maximum slope picking usually gives a pick close to the fifty-percent amplitude threshold pick. The crosscorrelation pick is the most sensitive to secondary energy and gives the least accurate pick at all frequencies.

All of threshold picks are insensitive to secondary energy arriving later than about one-quarter period of the dominant frequency ( $\mathrm{T} / 4$, the time from zero to peak) after the true first break. Thus they are all accurate to better than one-half of the dominantfrequency Fresnel zone half-period criterion (T/2). In contrast, the cross-correlation is 
inaccurate at about the Fresnel zone criterion because energy from the second arrival interferes with the first arrival for delays up the entire length of the wavelet. These differences demonstrate that the dominant-frequency half-period Fresnel zone criterion may not relevant for all picking methods.

The erroneous pick time at very low frequency is the average of the two arrivals. If the arrivals were of different amplitude, the erroneous pick would be a weighted average. Thus, even when the pick is very inaccurate and the first arrival is small, the pick is always different from the pick where the object is absent.

\section{Motivation}

The initial exercise above suggests that the factors affecting complex traveltime picking accuracy and ultimately tomography resolution are neither simple nor straightforward. Interference of wavelets with different frequency and amplitude content introduces another degree of complexity to the problem. Cerveny et al. (1992), Sheng et al. (2003), and Dahlen et al. (2004) conducted studies of a single velocity anomaly in an otherwise homogeneous medium on a propagating plane wave to examine the manner and extent of interference between the diffracted, transmitted, and direct energy upon the recorded ground shaking at the receivers. Nolet et al. (2000), Hung et al. (2001), and Marquering et al. (1999) extended this theme by constraining the single anomaly to be small and circular or spherical. The following sections further test these concepts with synthetic data with a causal wavelet. The accuracy of traveltime picks of various picking methods are first tested for noise-free data in the presence of a small object. These picks are then used in traveltime tomography to test theoretical detection and resolution of the small object and to compare the final images produced by the different picking criteria. 
Noise is then added to the seismic data to test the statistical effect upon the picks. The effects of noise reduction methods upon the picks are also tested. Finally the noisy picks are inverted to determine the practical detection and imaging limits of tomography for small objects.

\section{Synthetic Data}

We embedded a single circular object within a homogeneous velocity model (Figure 4). The object is in the center of a circle of receivers at one-degree spacing, and a single shot replaces one of the receivers. The diameter of the shot-receiver circle is twenty times the diameter of the object at its center.

Synthetic acoustic seismic trace data were computed in two dimensions using eigenfunction expansion in the frequency domain (Imhof, 1996). The incident wave is decomposed into cylindrical waves with different frequencies and different angle orders. Each frequency and order can only forward- and back-scatter (transmit and reflect) into a cylindrical wave with equal frequency and order. The reflected and transmitted waves are combined by weighting each frequency and angular order with a frequency and order dependent scattering coefficient. Data were generated for a single source and 21 receivers at $1^{\circ}$ spacing from $160^{\circ}$ to $180^{\circ}$ around the circle from the source, and by symmetry 20 more receivers to $200^{\circ}$ (receivers highlighted in Figure 4). These 41 receivers will be referred to as the "transmission" receivers. At the other receivers around the circle, energy scattered from the object arrives more than a half-cycle behind the direct arrivals, and thus does not affect the threshold picks. Traveltimes at these receivers were calculated rather than picked from synthetic data. Due to the symmetry of 
the model, data from a single shot can be rotated in $1^{\circ}$ increments to simulate shots at every other position on the circle.

In a study with nearly identical models, Hung et al. (2001) found that the character of the waves at a receiver depends strongly on whether the object is faster or slower than the background model. For fast anomalies, the energy traveling through the object arrives earlier but the amplitude is reduced by defocusing. The width of a lowfrequency pulse is broadened by later arriving diffractions around the object. For slow anomalies, the first arrivals are the diffractions around the object. Their amplitude is increased due to ray focusing and the pulse width at low frequency is narrowed due to more coincident arrivals.

We set the velocity of the anomalous object such that the infinite-frequency first arrival time was advanced or delayed by roughly equal amounts relative to the homogeneous (object-absent) model. Anomalies with 5\% above and 10\% below the background velocity give similar time shifts. Synthetic Green's functions were generated for models with $-10 \%, 0 \%$ (homogeneous), and $+5 \%$ anomalies (Figure 5 ). The Green's functions were broadband for frequencies that cover the trace window shown in Figure 5 and up to Nyquist frequency using 80,000 samples. This bandwidth is much broader than the wavelets to be used. The direct arrivals for the fast anomaly and homogeneous velocity model show a large, sharp spike followed by a low-amplitude, low-frequency transient tail (Figure 6). The transmitted arrival through the slow anomaly shows a lowamplitude, low-frequency rise leading up to the large spike. The diffracted arrivals are not an impulsive spike, but are a small step followed by a low-frequency transient. The diffracted energy arrives before the transmitted arrival for the slow anomaly. 
The Green's functions were then convolved with the Berlage wavelet at a range of dominant frequencies that correspond to Fresnel zones ranging from much larger to much smaller than the object. The Fresnel zone diameters quoted are measured in the homogeneous background medium without the object present, while recognizing that the presence of the object would alter the Fresnel zone. Figure 7 shows a data comparison for the three velocity models for an object only 2.5 times larger than the dominant wavelength. The object is about one-third the size of the dominant-frequency Fresnel zone (Figure 4a). The difference in arrival times is clearly visible even though the object is much smaller than the dominant-frequency Fresnel zone. It would be difficult to imagine a picking method that could not detect this difference, even in the presence of moderate noise.

Figure 8 shows the $0^{\circ}$ receiver at a range of frequencies to illustrate interference between direct and diffracted arrivals at low frequency. Destructive interference begins where the delay between arrivals is within a period, but this does not affect picks of the first onset. Below a half-period, at which the object size equals a Fresnel zone boundary $(2 \mathrm{r} / \mathrm{FZ}=1)$, constructive interference begins to affect the tail of the first peak. Below about a quarter-period, it affects the shape of the wavelet before the first peak, where threshold picks are made. For the fast model, the first peak shifts later in time at low frequency. The slow model shows the same general broadening, but the first peak develops two lobes whose separations shrinks to a single peak at the lowest frequencies. 


\section{Noise-free Picks for Small Objects}

The synthetic data were picked at a broad range of wavelet dominant frequencies using the picking methods described above. To compare different picking methods, the picks were corrected for the time delay of that pick using the reference wavelet, as described above. In this manner, if the observed signal equals the reference wavelet (if the Green's function is a single delta function) then all the picking methods give the same infinite-frequency pick time. For the receiver at $0^{\circ}$, directly behind the object, Figure 9 shows the corrected picks using the threshold, first peak, maximum slope, and known wavelet cross-correlation picking methods.

For the fast object, frequencies where the object size is larger than $\sim 75 \%$ of the size of the dominant-frequency Fresnel zone give picks that match the infinite-frequency times. This means that the waveform of the direct first arrival at those frequencies, though smaller in amplitude due to ray defocusing effects, has the same shape as the pilot wavelet. The reason for this is simple: the time difference between the sped-up direct arrival and the diffracted arrivals is greater than the length of the wavelet. The fast object has shrunk the size and altered the shape of the direct first arrival's Fresnel zone so that diffracted waves are no longer within it. When the object shrinks to below eight-tenths the size of the Fresnel zone (or the Fresnel zone grows due to a decrease in frequency), the diffracted arrival destructively interferes with the later part of the first arrival and the cross-correlation picks are slightly earlier in time (Figure 9). Similar shifts occur for the threshold picks, but for smaller objects / lower frequencies. Only when the object is less than $\sim 35 \%$ of the size of the dominant-frequency Fresnel zone do all of the picks start to differ significantly from the infinite-frequency time, shifting towards later times (Figure 
9). Constructive interference of the diffracted energy broadens the recorded pulse and shifts the first peak and its ramp up to later times. Though all picking methods give late times at very low frequency, the picks are always different from the pick when the object is absent.

For the slow anomaly, the first arriving energy diffracts around the anomaly and the direct energy arrives later. Even at the highest frequencies, the picks fall later in time than the infinite-frequency pick (Figure 9b) because the Green's function for the diffracted energy is not impulsive and has a significant tail. At the point where the time delay between arrivals equals the time delay of the uncorrected pick, all picks rise toward later times. This occurs at different frequencies for different picking methods. The sharpness of this rise is due to the larger amplitude of the secondary arrival. Towards even lower frequencies / smaller objects, all the picks decrease to earlier times as the wavelength approaches the object size.

For both fast and slow anomalies, all of the picking methods are accurate for objects much smaller than the dominant-frequency Fresnel zone (Figure 9). Though the accuracy suffers, all of the picking methods detect the presence of the object down to where the object size is of the order of the wavelength (Figure 9). The pick is never equal to the pick with the object absent. Picking methods that use only the earliest part of the wavelet perform better than those that use later parts. Therefore the $10 \%$ threshold pick is the most accurate, higher thresholds up to the first peak are progressively worse, and the cross-correlation is worst. 


\section{Noise-free Tomography of Small Objects}

The Fourier Projection-Slice Theorem states that a plane-wave projection through an object provides a slice of the object's spatial spectrum (e.g. Averbuch et al., 2006). The application to seismic traveltime tomography [Mersereau et al., 1974; Rector et al., $1994]$ is complicated by dependence of the ray upon the velocity, but the theory can be generalized. When one takes the spatial Fourier transform of the measured forward scattered fields (an array of seismometers), one can achieve a slice of the 2-D spatial Fourier transform of the object. By illuminating the object from many angles, the whole 2-D or 3-D Fourier transform of the object can be reconstructed. Effectively, the theorem states that if a perturbation in time due to a small object can be detected, then the object can be tomographically imaged.

In Figure 9, accurate traveltime picks are obtained for objects much smaller than the dominant-frequency Fresnel zone, and inaccurate but detectable traveltime anomalies different from the background model are observed even for the smallest objects. The Projection Slice Theorem says that these very small objects can be imaged by tomography. The inaccurate picks at low frequency will degrade the resolution of the image, but the small object can be properly located by such data.

Traveltimes were picked from the noise-free data for all receivers from $160^{\circ}$ to $200^{\circ}$. Analytic infinite-frequency times were calculated for the other receivers in Figure

4. Picks were obtained for a range of frequencies using the $50 \%$ threshold and the crosscorrelation picking methods, correcting the picks for the known wavelet and picking method. The frequency range spans from the object being 1.6 times to 0.1 times the size of the dominant-frequency Fresnel zone. Infinite-frequency picks were also simulated. 
The picks were then rotated around the model circle to simulate shots and receivers at $1^{\circ}$ increments. Picks were made for both the slow and fast anomalous objects.

Each set of picks was inverted using the traveltime algorithm of Hole [1992]. This algorithm linearizes the nonlinear relationship between velocity and traveltimes by assuming only small perturbations to the velocity field. The linearization assumption is that these small perturbations do not significantly affect the ray path. Traveltimes and the ray paths are calculated using a 3-D finite-difference solution to the infinite-frequency eikonal equation [Vidale, 1990; Hole et al, 1995]. A gridded velocity field is defined as a starting model. Traveltimes are propagated away from a source to every grid node in the model. By following the gradient of the traveltime isochrones, the ray paths may be traced back from the receivers to the source. Using simple back-projection for the inversion, the misfit between the observed and calculated traveltimes is distributed over each grid cell intersected by the ray path, where each cell receives a slowness (the inverse of velocity) perturbation equal to the total misfit divided by the length of the ray. The slowness perturbation for each grid node is computed from a ray-length dependent weighted average of rays in a volume of cells surrounding the grid point. Finally, a moving average filter of some previously determined size is passed over the slowness perturbation field and added to the starting slowness model. The process is repeated, forward-modeling at each step, to converge to a solution of the nonlinear problem.

The model measures $521 \times 521$ cells with a grid spacing that is $4 \%$ of the diameter of the anomalous object. All the inversions start with the correct homogeneous background velocity. The initial iterations were strongly smoothed to 300 cells. After the third iteration, the smoothing sizes were reduced to two-thirds of the previous size 
and the process was repeated to progressively smaller smoothing sizes. Additional iterations were used at the finest smoothing scales. This approach strongly reduces the effect of the starting model and pushes the model towards a very smooth structure. In this case, the background model is correct, the ray coverage is very dense, and the data are accurate, so changes to the model occur in only the last iterations with smoothing similar to or smaller than the object size. However, the large smoothing is needed for later tests with noisy data and/or a smaller subset of rays. Due to the excellent ray coverage, the finest scale below which artifacts are introduced with no addition of relevant structural detail occurred at a smoothing size $\sim 1 / 4$ of the size of the object. This limit is due to gaps in first-arrival ray coverage that are caused by the object (Figure 10).

Figure 11 shows sample tomograms. Even at infinite frequency, tomography images a slightly larger object in the fast case, and slightly smaller in the slow. This is due to ray focusing and defocusing, which creates gaps in the first-arrival ray coverage, and smoothing during the inversion. For noise-free data, the anomalies image as smooth, symmetrical, circular features where the radius of the object image is a function of frequency and the picking method. Since the image is symmetrical, the full-width, halfmaximum amplitude is easy to pick in each tomogram (Figure 12). Generally, the imaged anomaly broadens at very low frequency. The frequencies where the picks become inaccurate (Figure 9) correspond to the frequencies where the image broadens (Figure 12), a direct cause-and-effect relationship.

Some existing literature states that traveltime tomography cannot image objects smaller than a Fresnel zone [e.g. Marquering et al., 1999; Gundmundsson, 1996; Baig et al., 2003; Williamson, 1991; Snieder et al, 1996]. The relevant frequency is usually 
indicated or inferred to be the dominant frequency in the data. Figure 11 indicates that objects much smaller than the dominant-frequency Fresnel zone can be accurately picked and imaged. Very small objects approaching the size of the wavelength can be detected to produce a lower-amplitude smoothed image. The relevant frequency for the Fresnel zone may be the maximum frequency in the data. For the noise-free, causal data above, the maximum frequency is the Nyquist sampling frequency; in principle the first non-zero sample could be picked (in practice the synthetic data contain some numerical noise). In realistic noisy data, the maximum usable frequency is less than infinite. A natural guess might be that the maximum frequency is that at which the noise exceeds the signal. However signal does exist at higher frequencies below the noise and may be statistically extractable. The next sections test the effects of noise on picking and tomography.

\section{$\underline{\text { Noise and Single Trace Picking }}$}

It is possible to pick and successfully invert noise-free traveltime data for objects as small as a fraction of the dominant-frequency Fresnel zone. Realistic data, however, are noisy and will result in less accurate picks and tomographic inversion. Noise was added to the synthetic data to assess the effects on picking accuracy and to investigate methods of improving signal-to-noise ratio (S/N). Random, white, Gaussian noise was added to the data using the Seismic Unix utility [Stockwell et al., 2007]:

$$
\begin{aligned}
& \text { output }=\text { signal }+ \text { scale } * \text { noise } \\
& \text { scale }=(1 / a) *(|b| / \sqrt{2}) / \sqrt{c}
\end{aligned}
$$


where $a$ is the user defined $\mathrm{S} / \mathrm{N}, \mathrm{b}$ is the maximum amplitude in the signal, and $\mathrm{c}$ is energy per sample. The most common definition of $\mathrm{S} / \mathrm{N}$ is framed in terms of the power spectral density ratio of signal versus noise rather than the amplitudes as in the above equation.

The degree of wavelet interference between arrivals can significantly affect the maximum amplitude of a trace. In order to ensure "consistent" noise from trace to trace the $0^{\circ}$ trace of synthetic data for the homogeneous (no object) model was used as a consistent reference level for parameters b and c. Different levels of noise were added to the synthetic data (Figure 7) at a single frequency for both the fast and slow anomaly (Figure 13). The dominant frequency corresponds to an object $\sim 0.35$ times the size of the Fresnel zone.

Two thousand different noise realizations were added to the $0^{\circ}$ seismic trace at each $\mathrm{S} / \mathrm{N}$. These traces were automatically picked using the $50 \%$ threshold criterion and corrected, and the statistics were investigated. Histograms of picks for a single trace with different noise realizations were constructed (Figure 14). The standard deviation of the distributions scales with approximately $[\mathrm{S} / \mathrm{N}]^{-1.0}$ (Figure 15). The mean of each distribution is approximately equal to the noise-free pick. This is an important point: even though the error on an individual traveltime pick may be too large to detect the presence of a small object, statistical averaging of many such picks may detect the object.

The stacking, or addition, of seismic traces for a repeated or very similar sourcereceiver pair is a common method for increasing the $\mathrm{S} / \mathrm{N}$. The common expression for $\mathrm{S} / \mathrm{N}$ (in terms of energy) improvement due to stacking says that the improvement is proportional to $\mathrm{N}^{0.5}$, where $\mathrm{N}$ is the fold, the number of traces in the stack. This translates 
to $\mathrm{N}^{0.25}$ in terms of amplitude. A large number of seismic traces were generated with different noise realizations and a $\mathrm{S} / \mathrm{N}$ of 2 . They were stacked at a range of folds and picked. This was repeated 2000 times and a histogram produced (Figure 16). The pick standard deviation improved proportional to $\sim \mathrm{N}^{-0.25}$ (Figure 17).

Next, the seismic traces were picked before stacking and groups of these picks were averaged at a range of averaging "folds" $\mathrm{M}$ (Figure 18). The results of averaging different realizations indicate that the pick standard deviation improves proportional to $\sim \mathrm{M}^{-0.5}$ (Figure 19).

Though not exactly to the same extents, stacking followed by picking and picking followed by averaging improve $\mathrm{S} / \mathrm{N}$ in similar ways. The statistics suggest that the pick averaging improves the $\mathrm{S} / \mathrm{N}$ to a greater degree than stacking for a given fold. This is important because tomography averages traveltime picks without repeating a shotreceiver pair. Therefore it may be possible to extract information from the statistics that is hidden by the noise in an individual pick.

Though the smallest amplitude threshold picking criteria are the most accurate for noise-free data, they become unstable in the noisier traces. Cross-correlation picking methods will remain relatively stable for most noise levels, though they are the least accurate. There is a tradeoff between stable (precise) picks and accurate picks with resulting accurate tomographic resolution.

\section{Tomography Inversion of Noisy Data}

Linearized inversion expresses the data as a linear combination of the model

parameters. The inversion solves the system of linear equations by damped least-squares 
or some other method that minimizes a norm of the model and/or the data misfit subjected to regularization (e.g. Menke, 1989 p.141). However one chooses to invert, the calculated model parameters will be a weighted average of the data. Traveltime tomography obtains local velocity model parameters by averaging many traveltime picks. Since each velocity model parameter is a weighted average of many picks, tomography is capable of extracting information that is statistically hidden in the individual picks. This is similar to directly averaging picks to improve their accuracy, as discussed in the previous section. Tomography was performed on the noisy data to illustrate this effect.

Random noise at several different $\mathrm{S} / \mathrm{N}$ was added to the 41 synthetic data traces (highlighted receivers of Figure 4) at a frequency corresponding to an object $\sim 0.35$ times the size of the Fresnel zone, and the data were picked using the 50\% threshold. The receivers outside of the transmission receiver arc have analytic traveltime picks to which noise was added using a standard deviation appropriate for the $\mathrm{S} / \mathrm{N}$ according to the scaling laws. This process was repeated 360 times with different noise to simulate sources at all receiver positions in Figure 4. These data were then inverted using the same tomography scheme (Figure 20). The initial large smoothing and gradual reduction were necessary to stabilize the inversions with high noise, and the final acceptable smoothing was larger. With 360 sources and receivers, an object is successfully recovered for the whole range of $\mathrm{S} / \mathrm{N}$. The images of the object are no longer circular, nor symmetrical, and the width of the imaged object is not consistent. Instead, the rootmean squared misfit of the final image from the known true velocity model is calculated using only grid cells within the circle of shots and receivers (Figure 21c). The misfit increases at low $\mathrm{S} / \mathrm{N}$ for both the fast and slow objects. 
At $\mathrm{S} / \mathrm{N}$ of 3 , the standard deviation of the errors on the traveltime picks (Figure 14) is comparable to the difference between the noise-free pick time and the objectabsent pick time. The presence of the object is barely detectable in individual picks. However, Figure 22 shows a sample tomogram of the slow object data at $\mathrm{S} / \mathrm{N}$ of 3 clearly imaged only moderately smeared as compared to the noise-free case. For the fast object data at $\mathrm{S} / \mathrm{N}$ of 2 , the picking errors (Figure 14) are much larger than the difference between object and no-object times. The presence of the object is not detected in individual picks, yet a distinct, smeared, circular object is imaged at the correct location (Figure 22). The results are only possible if tomography is averaging the picks to recover information statistically hidden within the noise. The broader anomaly is caused by the additional averaging (tomography smoothing) required to recover the object.

Ray coverage is an important factor affecting spatial resolution of tomography. All traveltime tomography algorithms use gridding, smoothing, and/or regularization to ensure that each model parameter is constrained by multiple rays. In the tomography discussed above, incredibly dense ray coverage ensured that Fresnel zone effects would be separable from ray coverage effects. To investigate ray coverage effects, the noisefree data were sub-sampled. This was accomplished by only including every second, third, fifth, tenth, fifteenth, thirtieth, and forty-fifth shot and receiver (Figure 21). The inversion scheme and smoothing strategy were the same as described above, but the gradual reduction in smoothing is more important here. In some cases, convergence had to be stopped at a larger final smoothing. For both fast and slow objects, the inversion produces identical or nearly identical images down to where the source and receiver number equals twenty-four, below which the imaged anomaly grows. This reduction in 
resolution occurs when the rays surrounding the object leave a space larger than the noray gap (Figure 10). The sparse ray coverage is then directly responsible for limiting the spatial resolution.

The noisy data were also sampled at a smaller number of sources and receivers (Figure 23). As ray density decreases, the image deteriorates much more rapidly than for the noise-free case (Figure 20). At the lowest number of sources and receivers and worst $\mathrm{S} / \mathrm{N}$, the inversion becomes unstable and does not successfully recover the object. The averaging of picks from densely sampled rays is clearly responsible for extracting information from the noise to image the object.

The previous section suggests that noisy picks statistically see the object in the same way (have the same mean) as the noise-free picks. This section suggests that tomography is capable of improving the statistics. This demonstrates that the relevant frequency for the Fresnel zone criterion is higher than the maximum frequency above noise in a single trace or the error on an individual traveltime pick, because tomography can extract information from beneath this noise. The limiting factors are the picking method, the shape of the wavelet, the noise level, the data density, and tomographic inversion non-uniqueness.

\section{Conclusion}

Ray theory has been a useful tool for many years in a broad range of applications. However, in recent studies it has been shown that the infinite-frequency approximation limits spatial resolution of the velocity model for realistic band-limited data. The limit in spatial resolution is usually described as the Fresnel zone, with the relevant frequency 
usually described or inferred to be near the dominant frequency in the signal. In most applications, the size of the dominant-frequency Fresnel zone is very large, often larger than the geologic features we wish to study. This work shows that the appropriate Fresnel zone criterion is not the dominant frequency. The relevant limit is associated with frequencies near the maximum in the data, and perhaps the frequencies of the signal hidden beneath the noise. More precisely, the limit is associated with the traveltime picking errors and the ability of tomography to improve these errors by averaging.

Traveltime picking of noise-free data is accurate for objects much smaller than the dominant frequency's Fresnel zone and detection is possible for objects smaller than the dominant wavelength. First-break picking methods that take advantage of the early part of the signal are more accurate (produce times closer to the infinite-frequency ray time) than cross-correlation methods which are more strongly affected by later-arriving energy. Noise lowers the accuracy of picks but they statistically average to equal the noise-free picks. Since tomography performs an averaging of picks from nearby rays, some information can be extracted from the noise for imaging. This inherent $\mathrm{S} / \mathrm{N}$ improvement can image objects that are undetectable in individual picks. Ultimately it is a combination of picking accuracy and ray coverage, and not the dominant frequency, that determines the spatial resolution that may be achieved.

The use of infinite-frequency modeling and the typical use of a single (usually first) arrival do limit the tomographic inversion. Methods that invert the full seismic waveform, including secondary and scattered arrivals, are capable of producing improvements in the velocity model by adding additional information and making better theoretical approximations. Further research on these methods is clearly merited. 
Testing more complex models which may include multiple small objects or small objects with irregular geometries could offer additional insight. However, the limits of traveltime tomography are often over-stated: objects much smaller than the dominantfrequency Fresnel zone can be recovered - and probably routinely are being imaged.

\section{$\underline{\text { References }}$}

Aldridge, D. F., 1990, The Berlage wavelet, Geophysics, v. 55, no. 11, p. 1508-1511

Averbuch, A., Coifman, R.R, Donoho, D.L., Elad, M., Israeli, M., 2006, Fast and accurate Polar Fourier transform, Applied and Computational Harmonic Analysis, no. 21, p. $145-167$

Baig, A.M., Dahlen, F.A., 2004, Statistics of traveltime and amplitude in random media, Geophysics Journal International, v. 158, p. 187-210

Bracewell, R.N., 1995, Two-Dimensional Imaging, Prentice-Hall, Inc., Englewood Cliffs, New Jersey, USA

Cerveny, V., Soares, J.E., 1992, Fresnel volume ray tracing, Geophysics, v. 57, n. 7, p. 902-915

Cohen, J.K., Stockwell, J.W., 2007, CWP/SU: Seismic Un*x Release No. 38: an open source software package for seismic research and processing, Center for Wave Phenomena, Colorado School of Mines

Dahlen, F.A., 2004, Resolution limit of traveltime tomography, Geophysical Journal International, v. 157 , p. $315-331$

Dahlen, F.A., Hung, S.H., Nolet, G., 2000, Frechet kernels for finite-frequency traveltimes-I. Theory, Geophysics Journal International, v. 141, p. 157-174

Gudmundsson, O., 1996, On the effect of diffraction on traveltime measurements, Geophysics Journal International, v. 124, p. 304-314

Hansen, S.E., Schwartz, S.Y., DeShon, H.R., Gonzalez, V., 2006, Earthquake relocation and focal mechanism determination using waveform cross correlation, Bulletin og the Seismological Society of America, v. 96, no. 3, p.1003-1011 
Hole, J.A., 1992, Nonlinear high-resolution three-dimensional seismic travel time tomography, Journal of Geophysical Research, v. 97, no. B5, p.6553-6562

Hole, J.A., Zelt, B.C., 1995 3-D finite-difference reflection traveltimes, Geophysics Journal International, v. 121, p.427-434

Hung, S.H., Dahlen, F.A., Nolet, G., 2001, Wavefront healing: a banana-doughnut perspective, Geophysics Journal International, v. 146, p. 289-312

Imhof, M.G., 1996, Multiple multipole expansions for elastic scattering, Journal of Acoustical Society of America, v. 100, no. 5, p. 2969-2979

Imhof, M.G., 2004, Computing the elastic scattering from inclusions using the multiple multipoles method in three dimensions, Geophysics Journal International, v. 156, p. $287-296$

Lin, G., Shearer, P., 2007, Estimating local $\mathrm{V}_{\mathrm{p}} / \mathrm{V}_{\mathrm{s}}$ ratios within similar earthquake clusters, v. 97, n. 2, p.379-388

Lindsey, J.P., 1989, The Fresnel zone and its interpretive significance, v. 8, n. 10, p. 3339

Marquering, H., Dahlen, F.A., Nolet, G, 1999, Three-dimensional sensitivity kernels for finite-frequency traveltimes: the banana-doughnut paradox, Geophysics Journal International, v. 137 , p. $805-815$

Mellors, R.J., Magistrale, H., Earle, P., Cogbill, A., 2004, Comparison of four moderatesize earthquakes in Southern California, v. 94, no. 6, p.2004-2014

Menke, W., Geophysical data analysis: discrete inverse theory., 1989, Academic Press., San Diego, California, USA

Nolet, G., Dahlen, F.A., 2000, Wave front healing and the evolution of delay times, Journal of Geophysical Research, v. 105, no. B8, p. 19043-19054

Pratt, R.G., 1999, Seismic waveform inversion in the frequency domain, Part 1: Theory and verification in a physical scale model, Geophysics, v. 64 , no. 3

Rector, J. W., Washbourne, J. K., 1994, Characterization of resolution and uniqueness in crosswell direct-arrival traveltime tomography using the Fourier projection slice theorem, Geophysics, v. 59, no. 11, p. 1642-1649

Richards, P.G., Waldhauser, F., Schaff, D., Kim, W., 2006, The applicability of modern methods of earthquake location, Pure and Applied Geophysics, v.163, no. 2-3, p.351-372 
Sheng, J., Schuster, G., 2003, Finite-frequency resolution limits of wave path traveltime tomography for smoothly varying velocity models, Geophysics Journal International, v. 152, p. 669-676

Snieder, R., Lomax, A., 1996, Wavefield smoothing and the effect of rough velocity perturbations on arrival times and amplitude, Geophysics Journal International, v. 125 , p. $796-812$

Spetzler, J., Sivaji, C., Nishizawa, O., Fukushima, Y., A test ray theory and scattering theory based on a laboratory experiment using ultrasonic waves and numerical simulation by finite-difference method, Geophysics Journal International, v. 148, p. $165-178$

Thore, P.D., Juliard, C., 1999, Fresnel zone effect on seismic velocity resolution, Geophysics, v. 64, n. 2, p. 593-603

Vidale, J. E., 1990, Finite-difference calculation of traveltimes in three dimensions, v. 55, n. 5 , p. 521-526

Williamson, P.R., 1991, A guide to the limits of resolution imposed by scattering in ray tomography, Geophysics, v. 56, no. 2, p. 202-207

Yang, H., Hung, S., 2005, Validation of ray and wave theoretical travel times in heterogeneous random media, Geophysical Research Letters, v. 32, L20302 


\section{Figures}

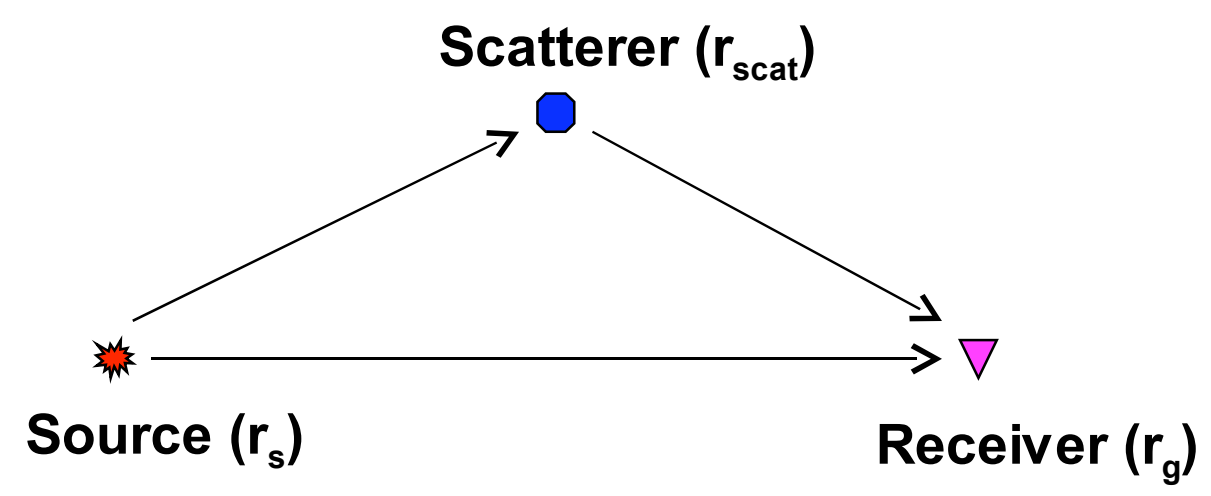

Figure 1. Raypaths from a source to a receiver for a uniform background velocity. A scatterer lies within the first Fresnel zone if the absolute value of the difference between the sum of the traveltime from the source to scatterer plus the traveltime from the scatterer to receiver and the direct traveltime from the source to receiver is less than one-half a designated period. 
(a)

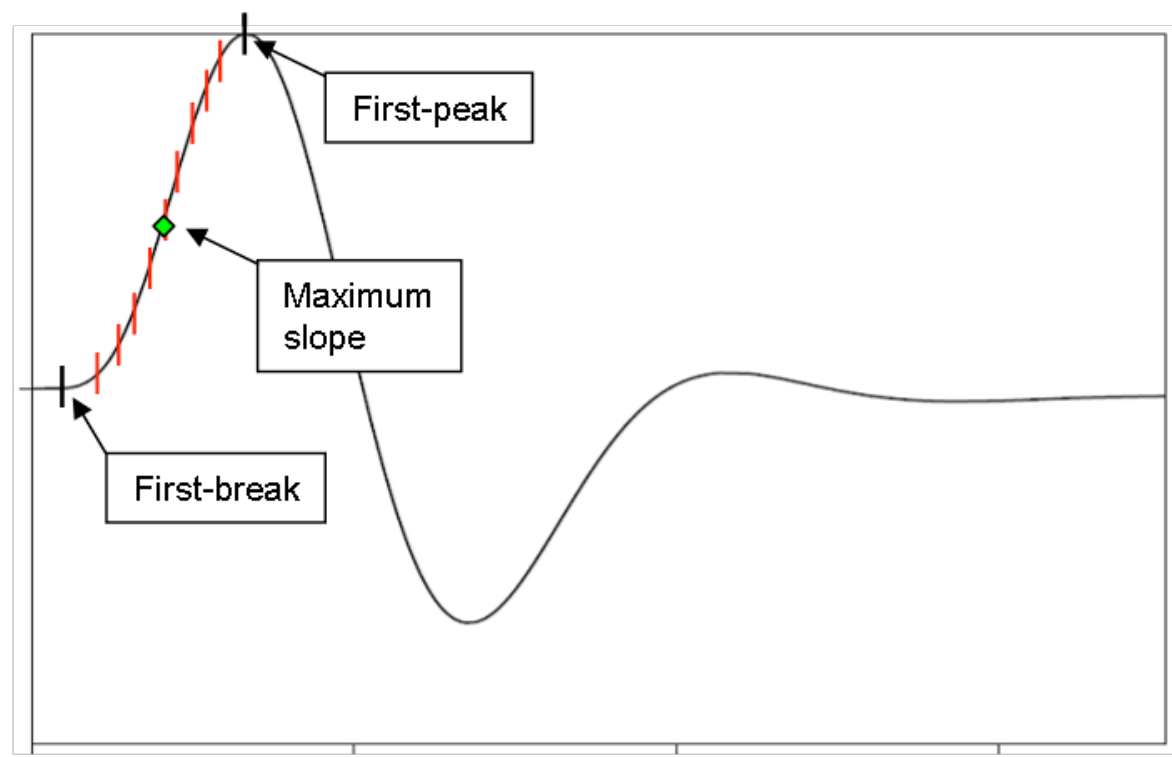

(b)

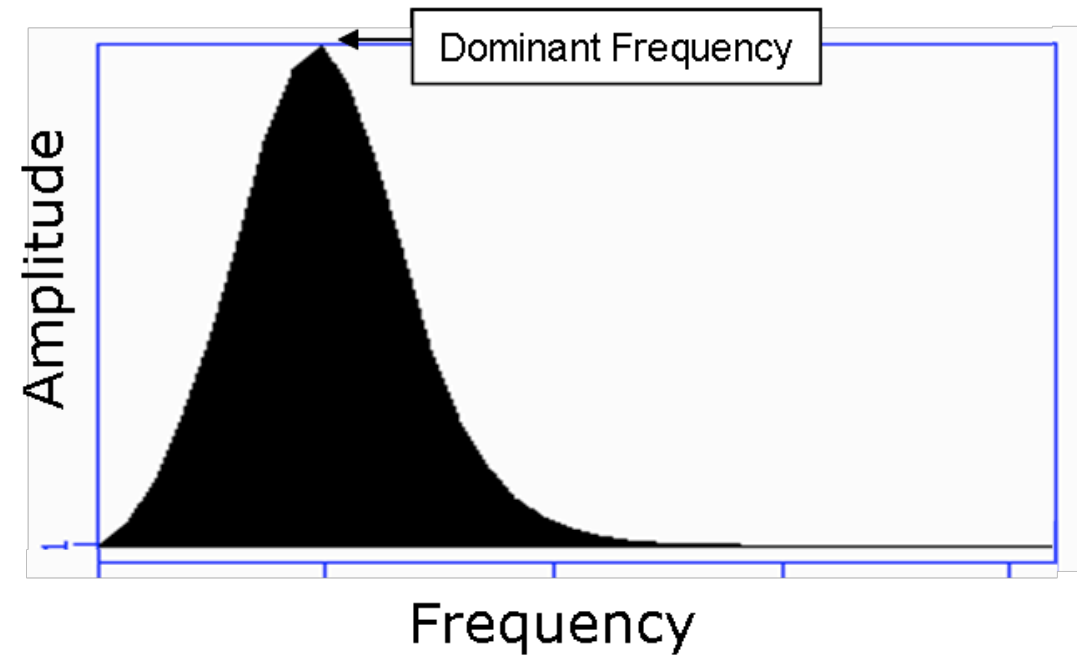

Figure 2. Berlage wavelet and its spectrum and illustration of picking criteria. (a) Red vertical bars represent percentages of the first-peak amplitude in 10\% steps. The firstbreak pick is the first non-zero sample in the signal. The maximum-slope pick is represented by the green diamond and lies close to the $50 \%$ amplitude threshold pick. 
(a)

(b)
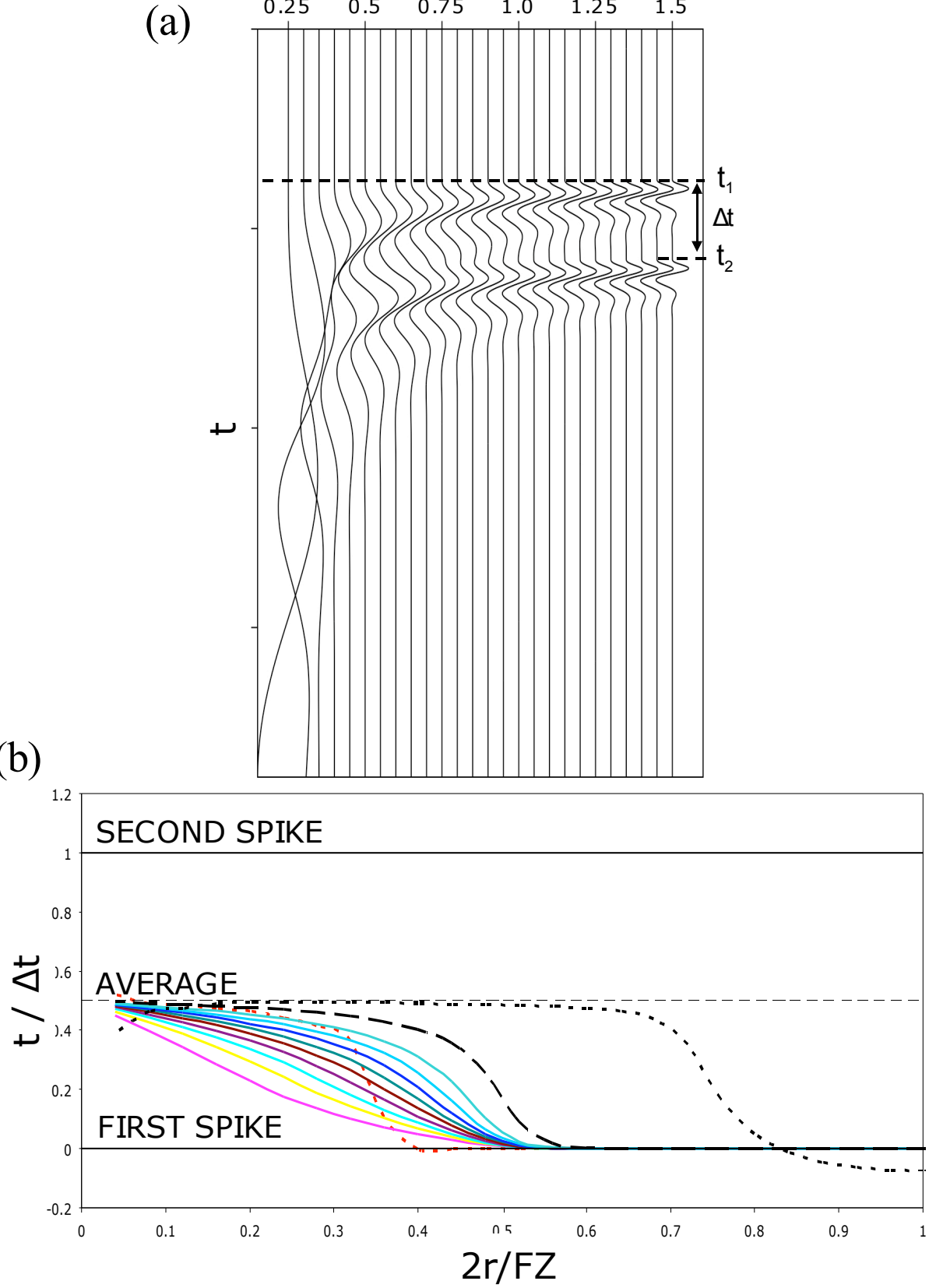

Figure 3. Two equal amplitude wavelets with decreasing frequency and increasing interference. (a) $\Delta t$ is the fixed delay between arrivals. (b) Picking results for above; arrivals adjusted as described in text. Amplitude thresholds are solid colored lines, 10\% pink and $90 \%$ blue; heavy dashed line first-peak; red dotted line is maximum derivative; black dotted line is cross-correlation with known wavelet. The "FIRST SPIKE" and "SECOND SPIKE" horizontal lines refer to infinite-frequency times of the two arrivals; the horizontal dashed line refers to the average position. 


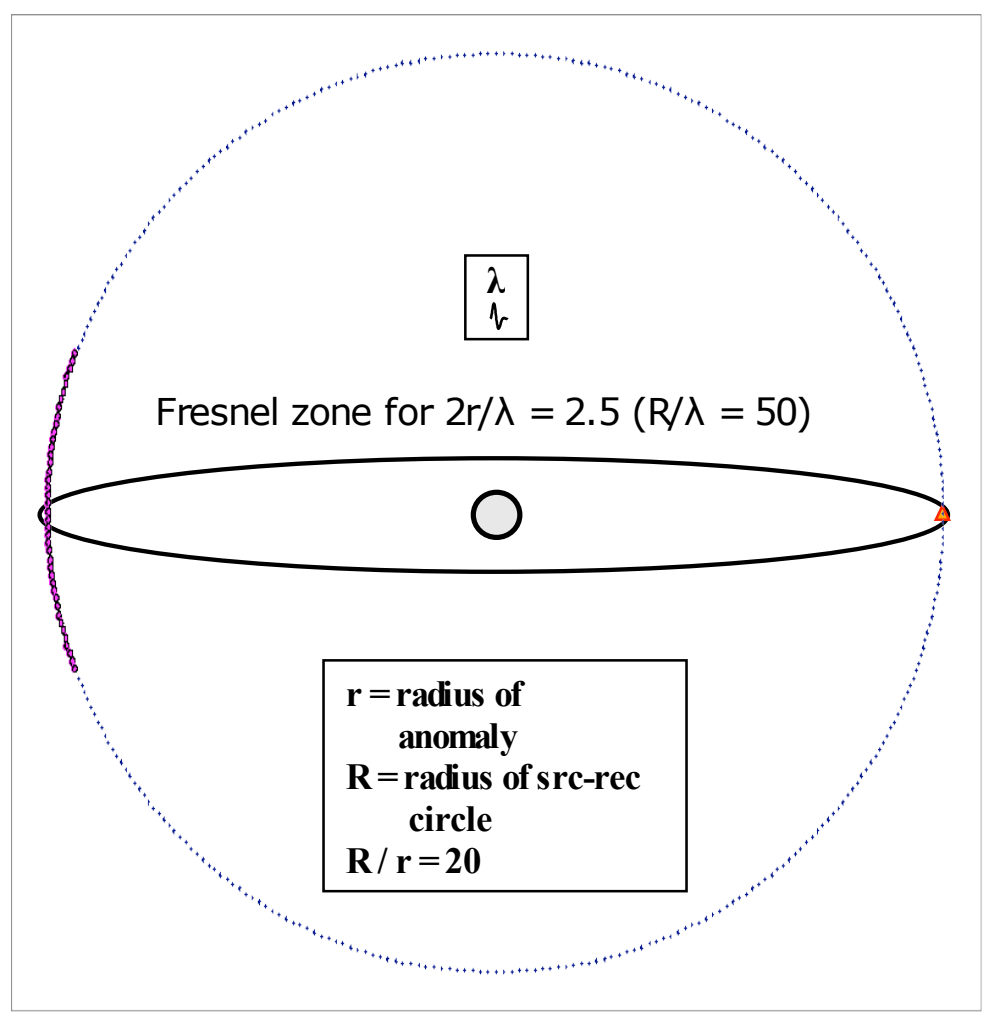

(b)
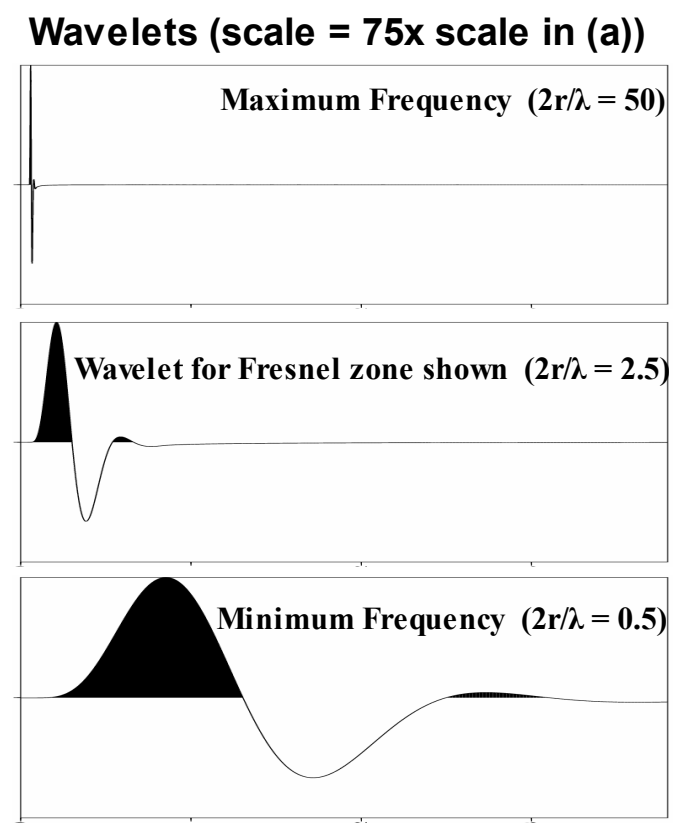

Figure 4. Model geometry. (a) Circle of 359 receivers in dots Green's functions were computed for a source at the red triangle for the 41 receivers highlighted on opposite side of the circle. Sample Fresnel zone is for a dominant wavelength $(\lambda) 2.5$ times smaller than the diameter of the anomaly. The dominant wavelength corresponds to a Fresnel zone three times larger than diameter of the anomaly. (b) The minimum, maximum, and sample wavelet corresponding to the Fresnel zone shown. Scale is $75 x$ that of(a). 


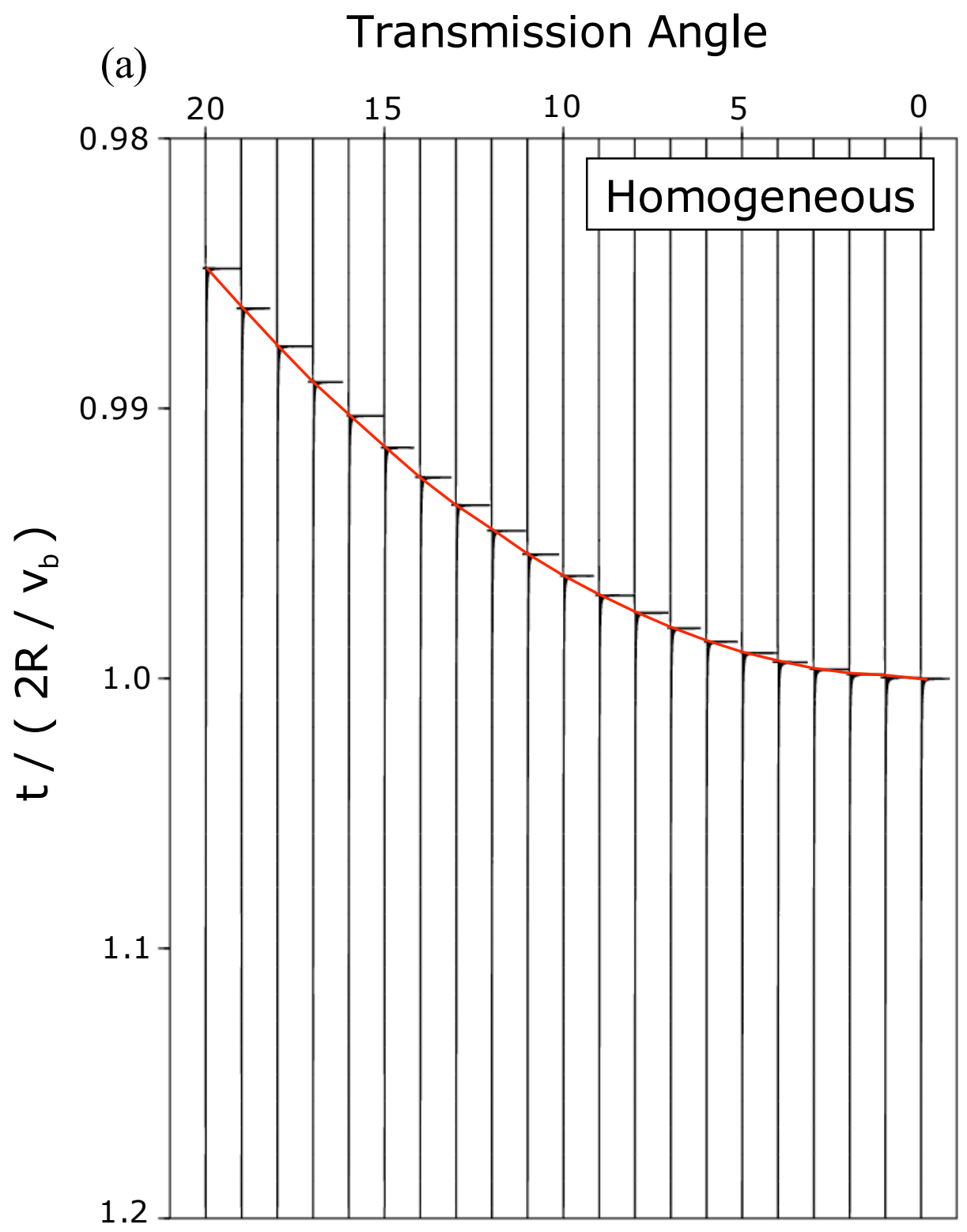

Figure 5a. (see caption on 5c) 


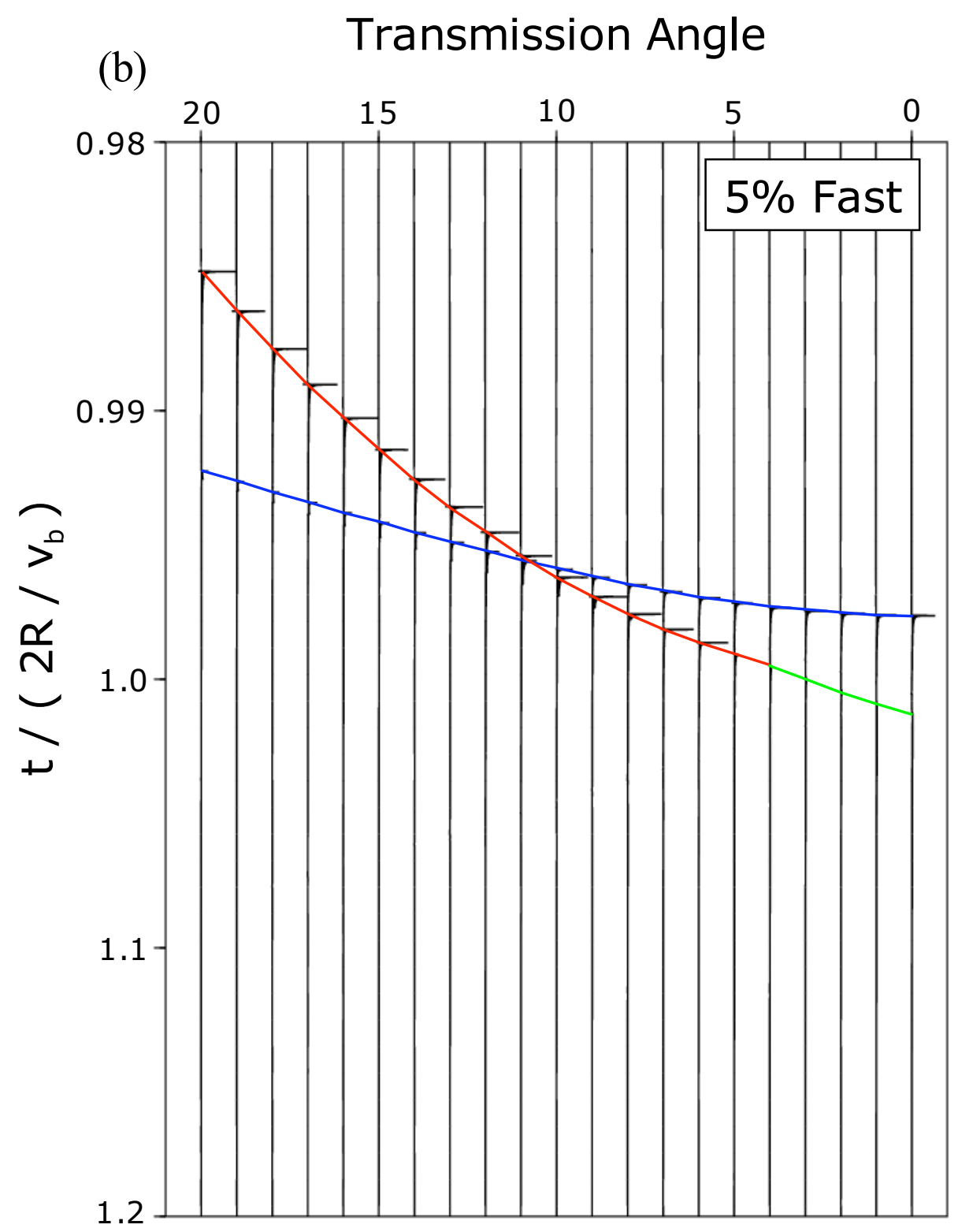

Figure $5 b$. (see caption on $5 c$ ) 


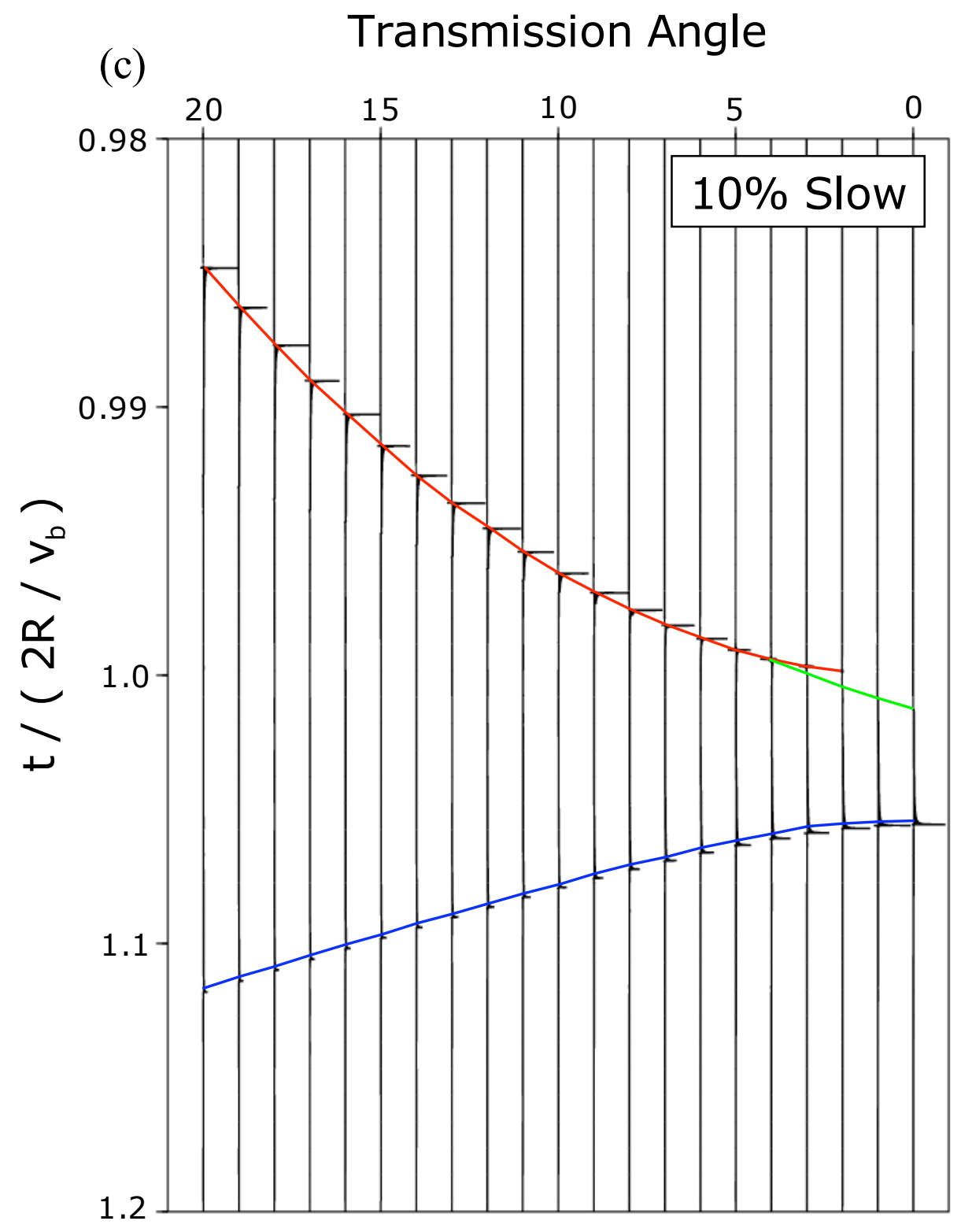

Figure 5c. Synthetic Green's functions for source and receivers highlighted in Figure 3. "Angle" is defined from the center of the circle in Figure 3, with 0 degrees the sourcereceiver line through the center of the object. $R$ is the radius of the receiver circle. $v_{b}$ is the background velocity. (a) The data with no anomalous object. The red line is the infinite-frequency picks. (b) Data for a fast object. The blue line is the transmitted energy through the object; the red line is the direct arrival; the green line is the raytime diffracted around the object. (c) Data for a slow object, with similar phases shown. 


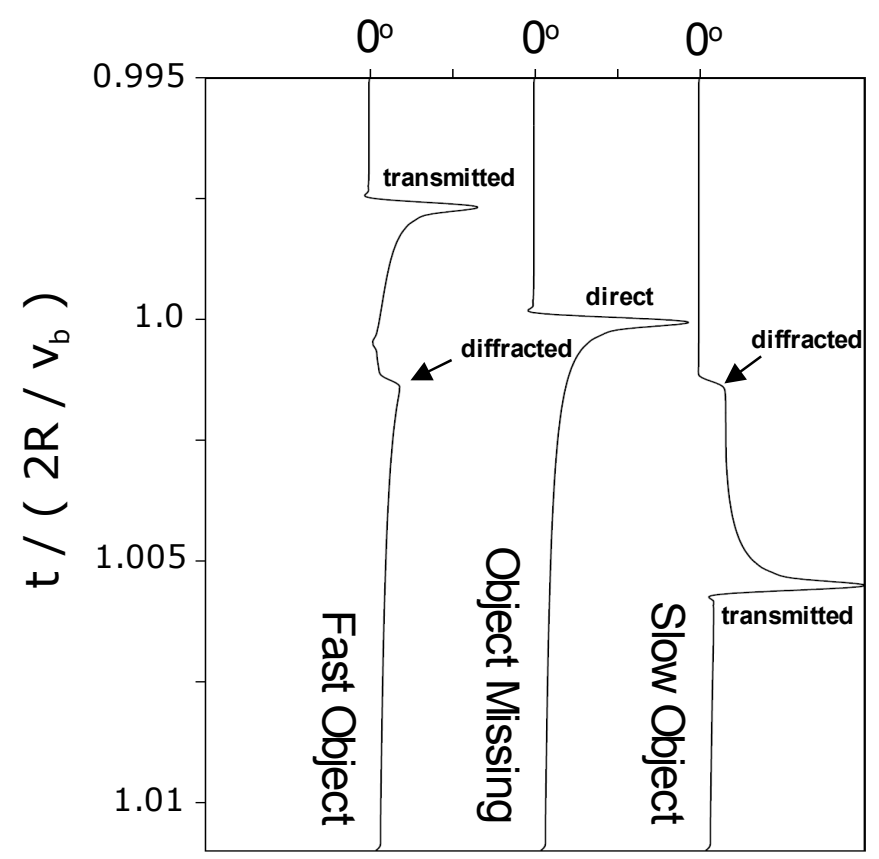

Figure 6. Green's functions for the 0 degree source-receiver pair expanded from Figure

5. Arrivals are labeled. 


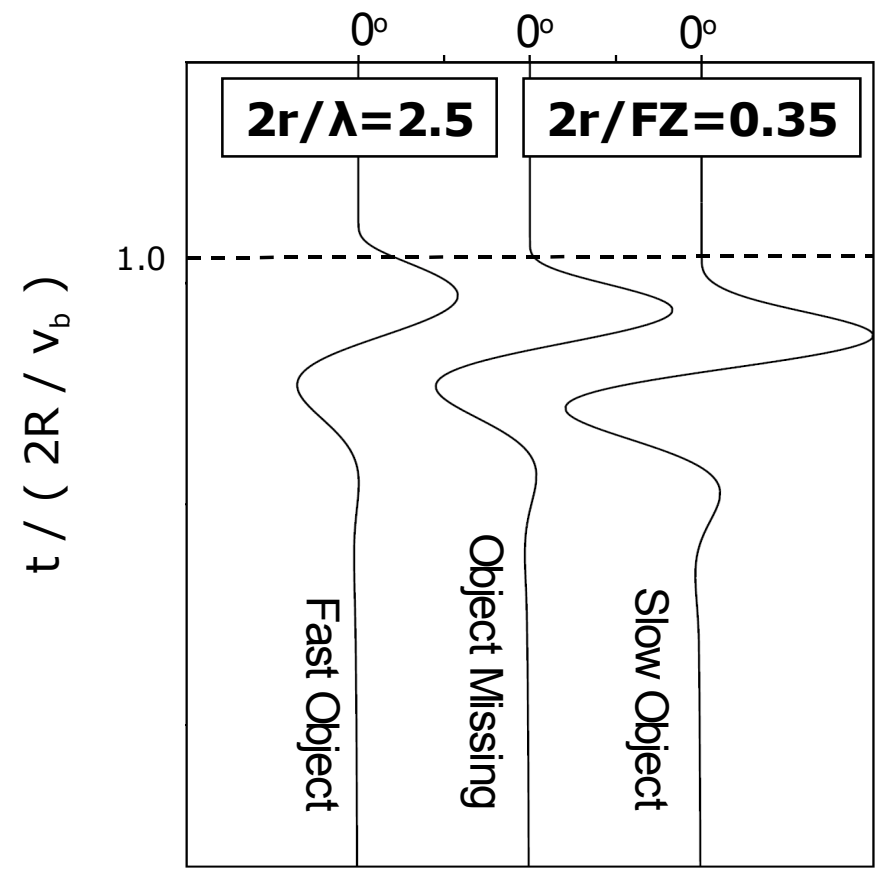

Figure 7. Seismic data created from Figure 6. The dominant frequency corresponds to the wavelength and Fresnel zone shown in Figure 4a. Dashed line marks first-break pick for the object missing trace. Different arrival times are easily observed even though the object is $1 / 3$ the size of the dominant frequency Fresnel zone. 

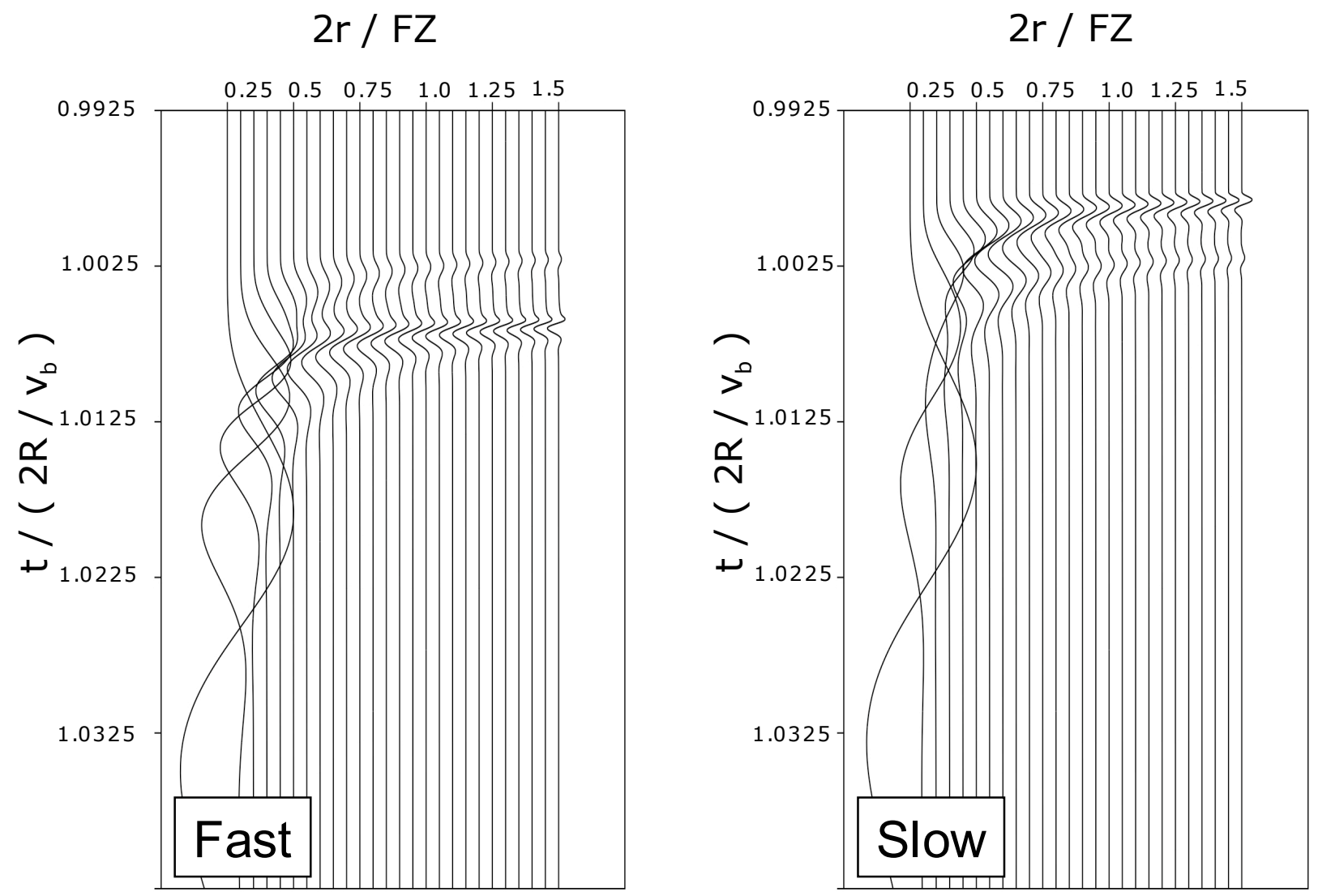

Figure 8 . Seismic data at a range of frequencies for the 0 degree source-receiver pair for (a) fast anomaly and (b) slow object. $2 \mathrm{r}$ refers to the diameter of the of the object, FZ refers to the width of the dominant frequency's first Fresnel zone. Dashed line is the infinite frequency first arrival (transmitted in (a) and diffracted in (b)). At no frequency is the wavelet the same as for the model with no anomaly. As the smallest Fresnel zones (highest frequencies) there is no interference, as the Fresnel zone increases (frequency decreases) the degree of wavelet interference increases. 


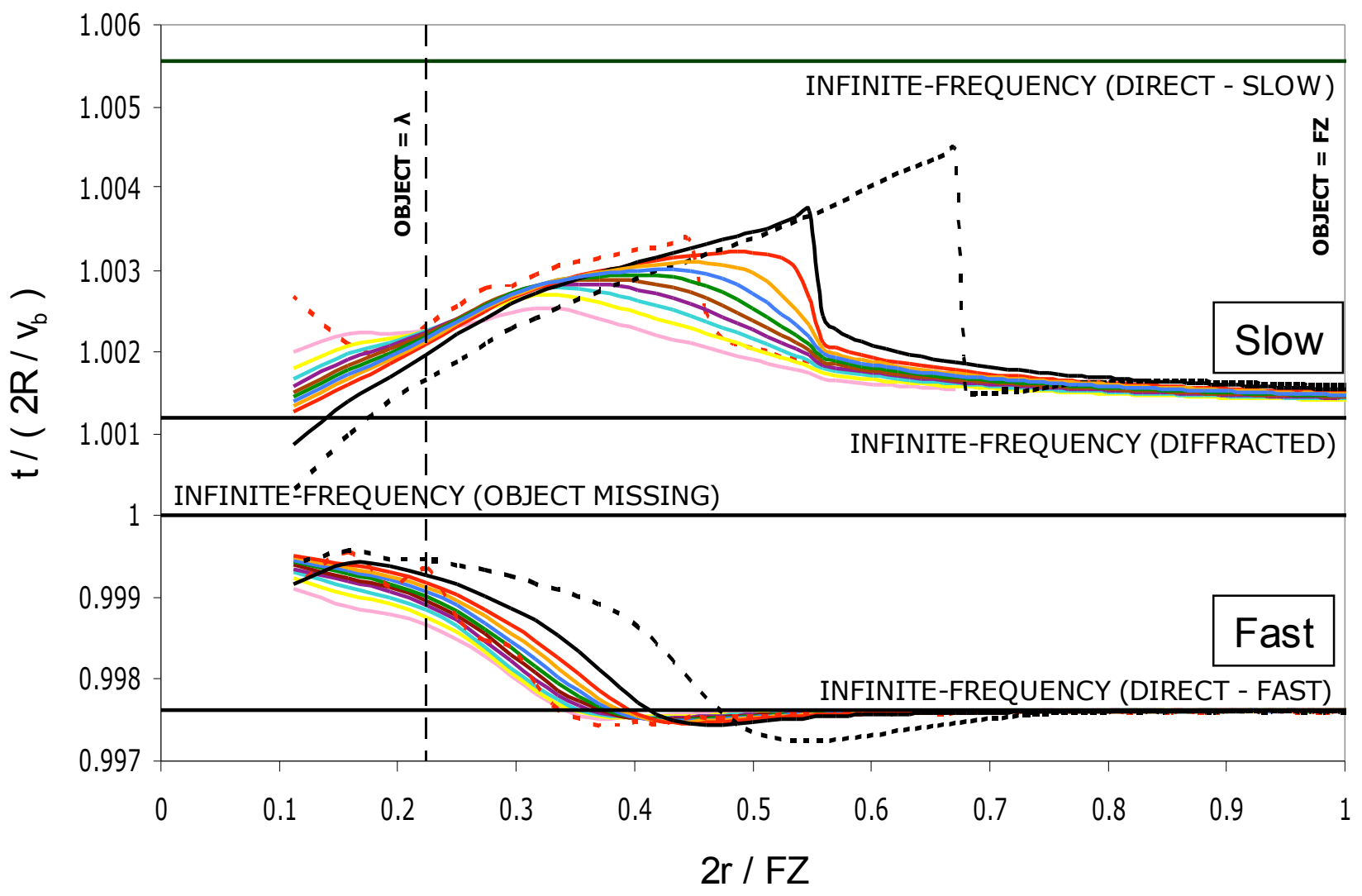

Figure 9. Picking results for noise-free data of Figure 8 for a fast object and a slow object as a function of frequency. Pick times adjusted as described in the text. $2 \mathrm{r}$ is the diameter of the object and FZ is the width of the dominant frequency's Fresnel zone. Amplitude threshold pick are solid colored lines, $10 \%$ pick in pink and $90 \%$ pick in blue; dashed line is the first-peak; dash-dotted line is maximum slope; dotted line is cross-correlation with known wavelet. 
(a)
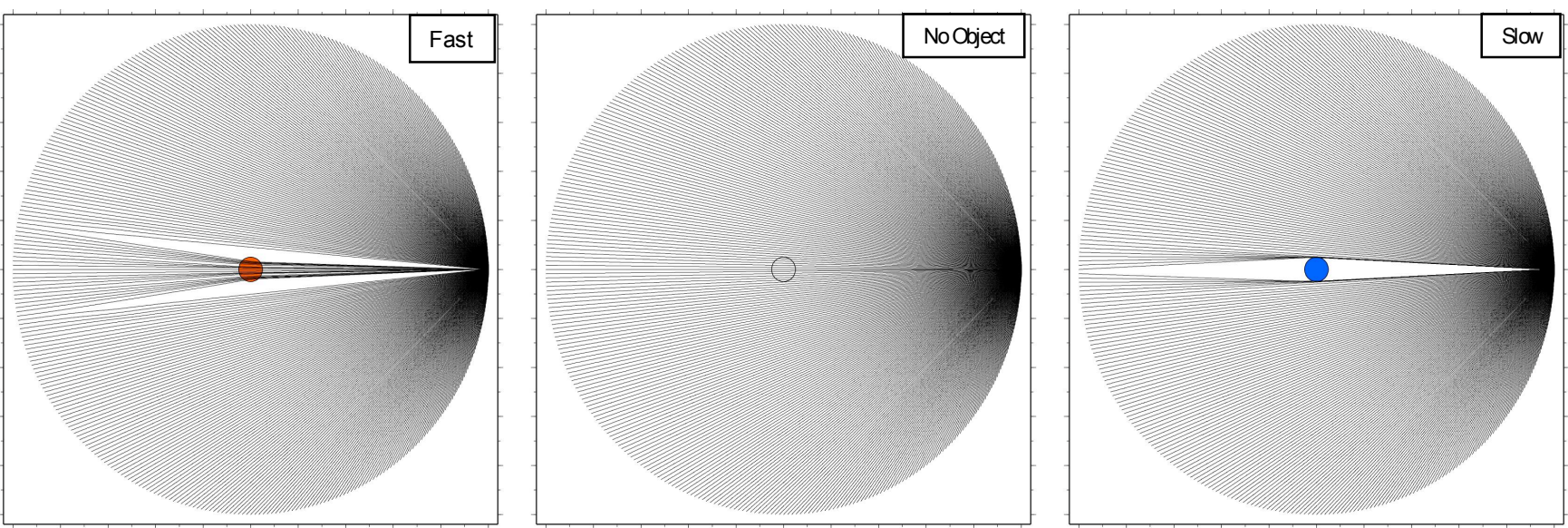

(b)
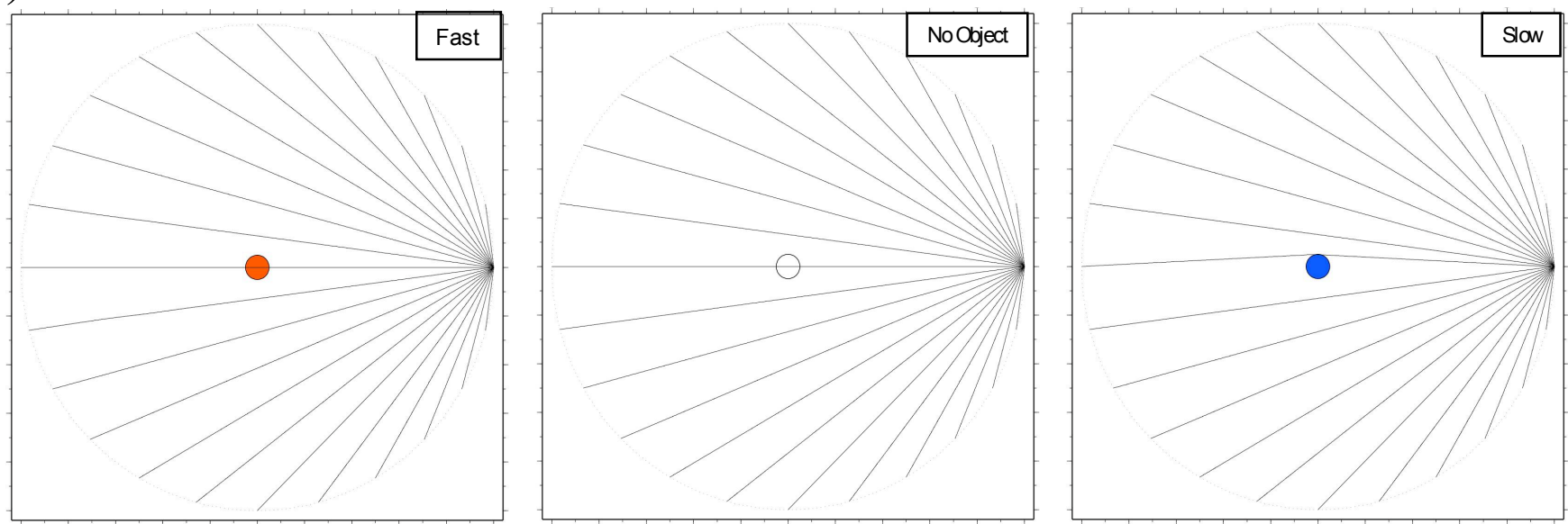

Figure 10. First-arrival ray tracing. (a) Ray tracing for a single source with 359 receivers with 1-degree spacing. (b) Ray tracing single source with 23 receivers with 15-degree spacing. 
Threshold Picks
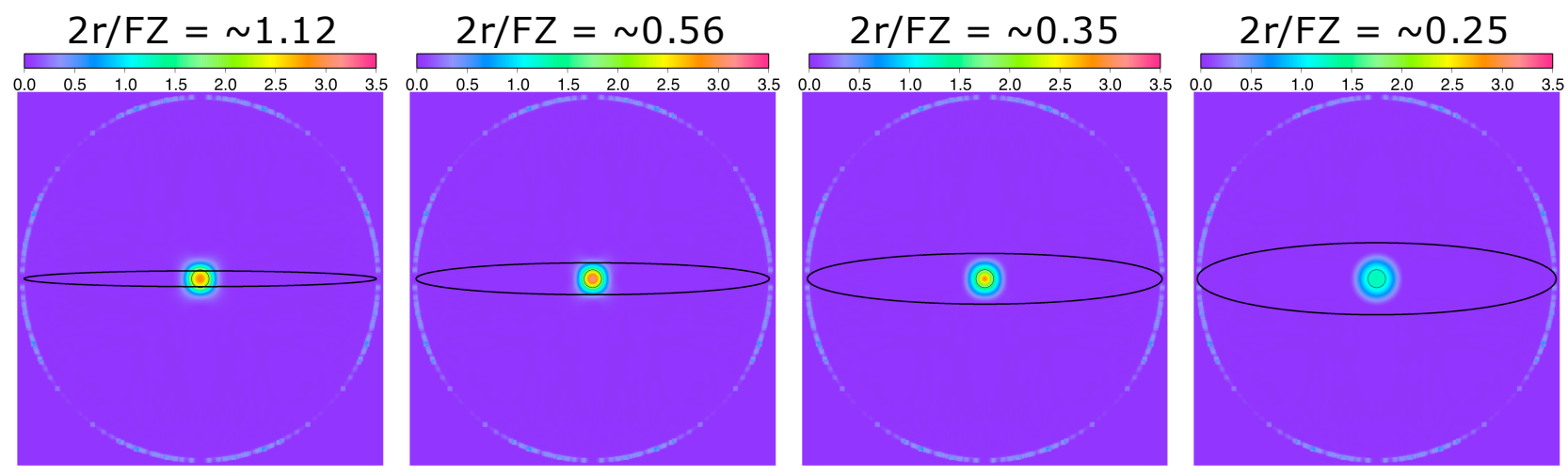

\section{Cross-correlation picks}
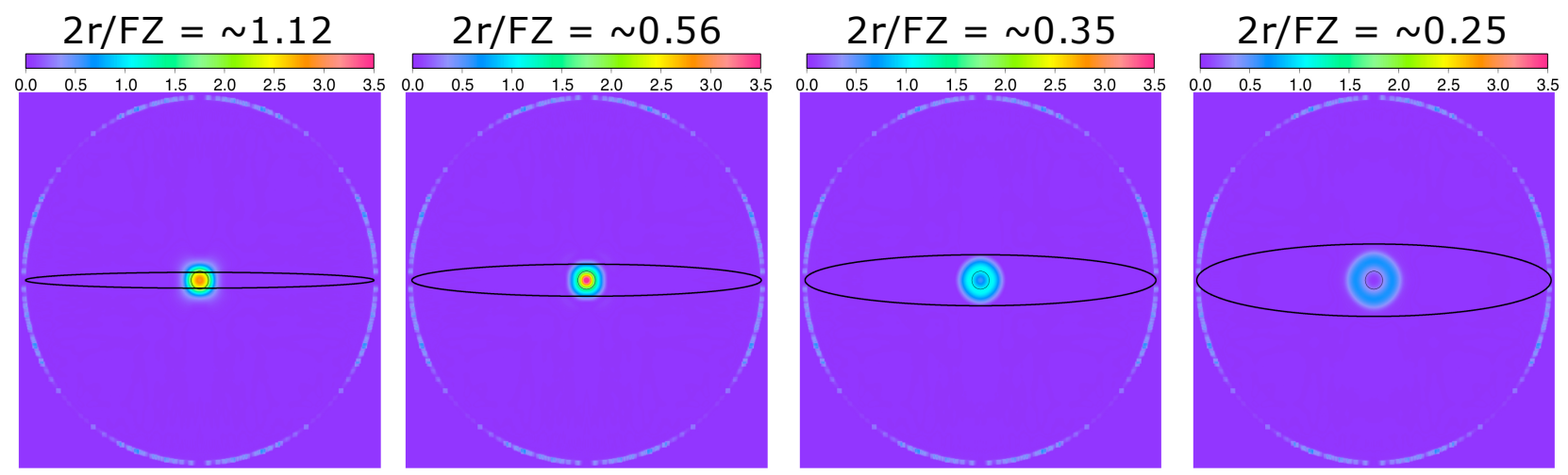

Figure 11a. (see caption on 11b) 


\section{Threshold Picks}
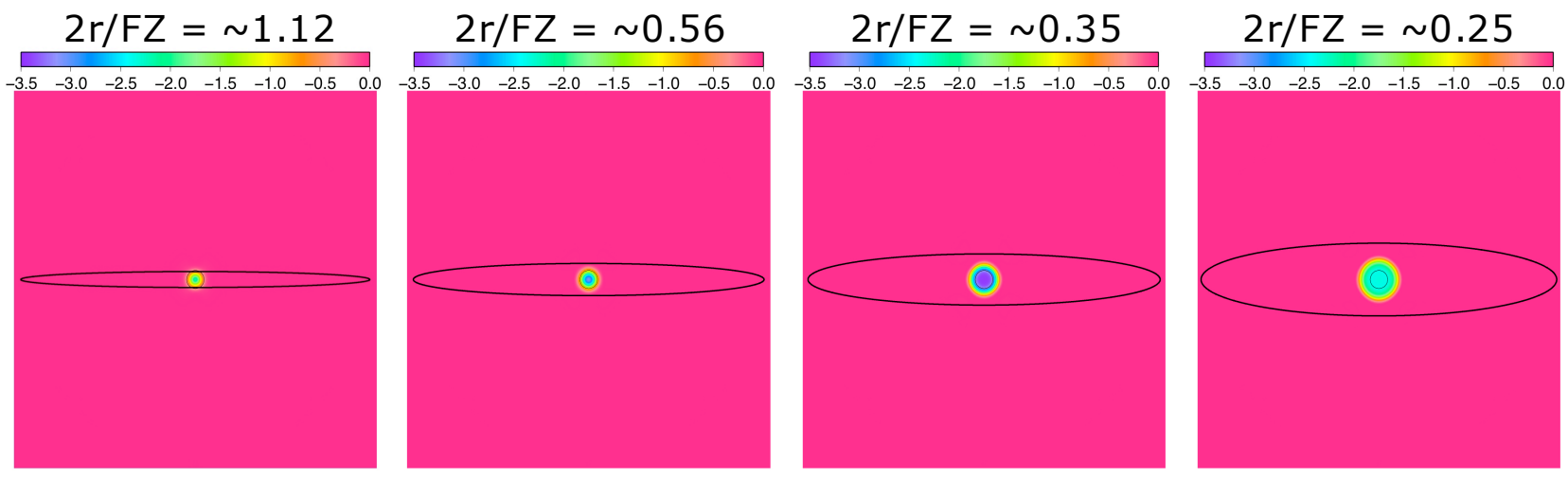

\section{Cross-correlation picks}
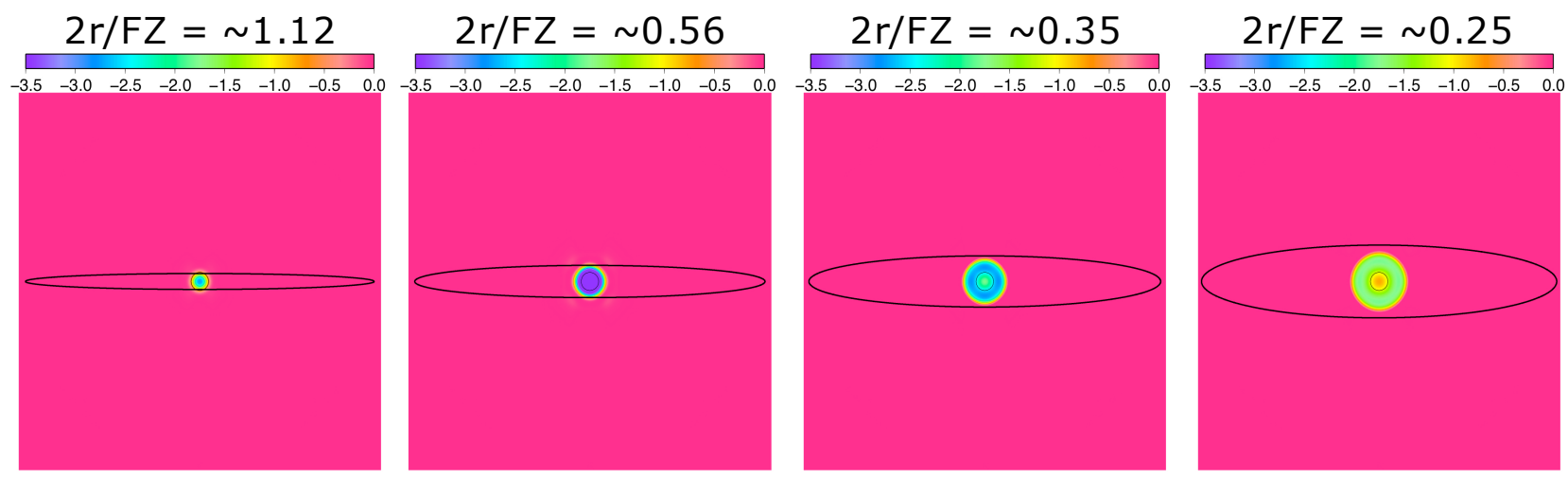

Figure 11b. Example tomograms for noise-free data, 360 shots and 360 receivers, and a range of frequencies. (a) Fast object 50\% threshold pick tomograms versus crosscorrelation with a known wavelet tomograms. (b) Slow object 50\% threshold pick tomograms versus cross-correlation with a known wavelet tomograms. Sample Fresnel zones for a single source-receiver pair illustrated for each dominant frequency. 


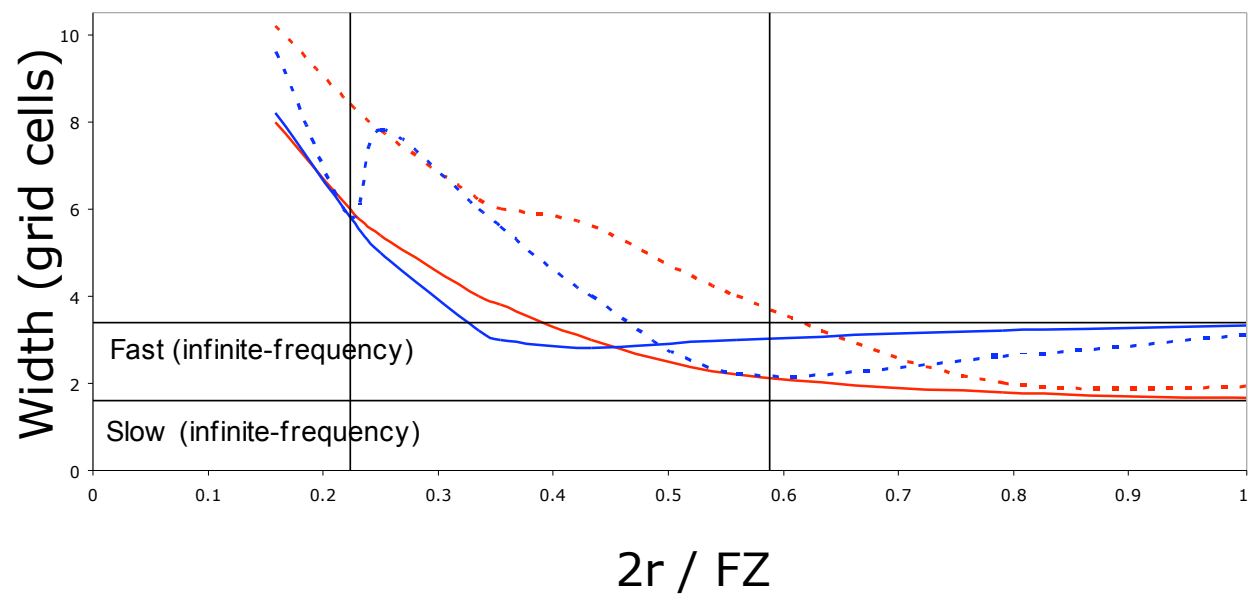

Figure 12. Width of anomaly resolved by tomography as a function of frequency for fast (blue) and slow (red) objects. Width is defined as the full-width at halfmaximum value. Tomography using 50\% threshold picks shown as solid lines, crosscorrelation as dashed. The width of the anomaly at infinite frequency is show for each model as solid, horizontal black lines; vertical black lines are the same as in Figure 9. An object is imaged for these noise-free tomograms for objects much smaller than the dominant frequency Fresnel zone. At very low frequencies, the object is less accurately resolved, but are still imaged at correct location. 


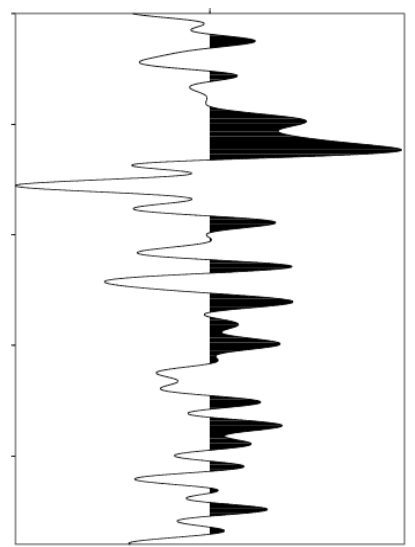

$\mathrm{S} / \mathrm{N}=2$

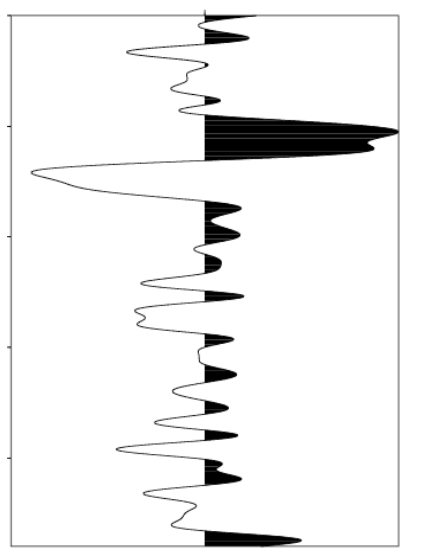

$\mathrm{S} / \mathrm{N}=5$

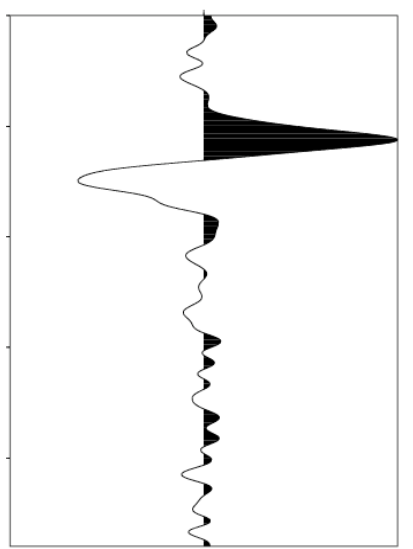

$\mathrm{S} / \mathrm{N}=15$

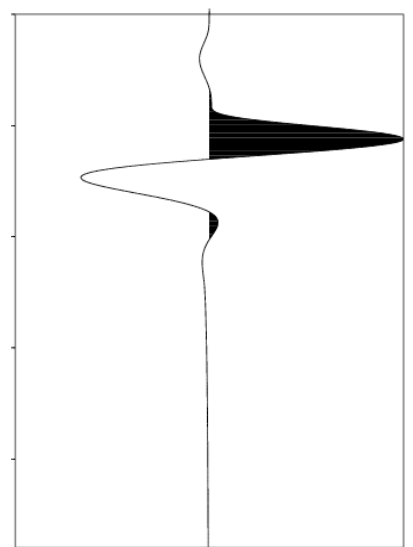

No noise

Figure 13. Example of the same trace with different $\mathrm{S} / \mathrm{N}$ added. The noise added is random, white, and Gaussian. "No noise" contains minor numeric noise. 
(a)

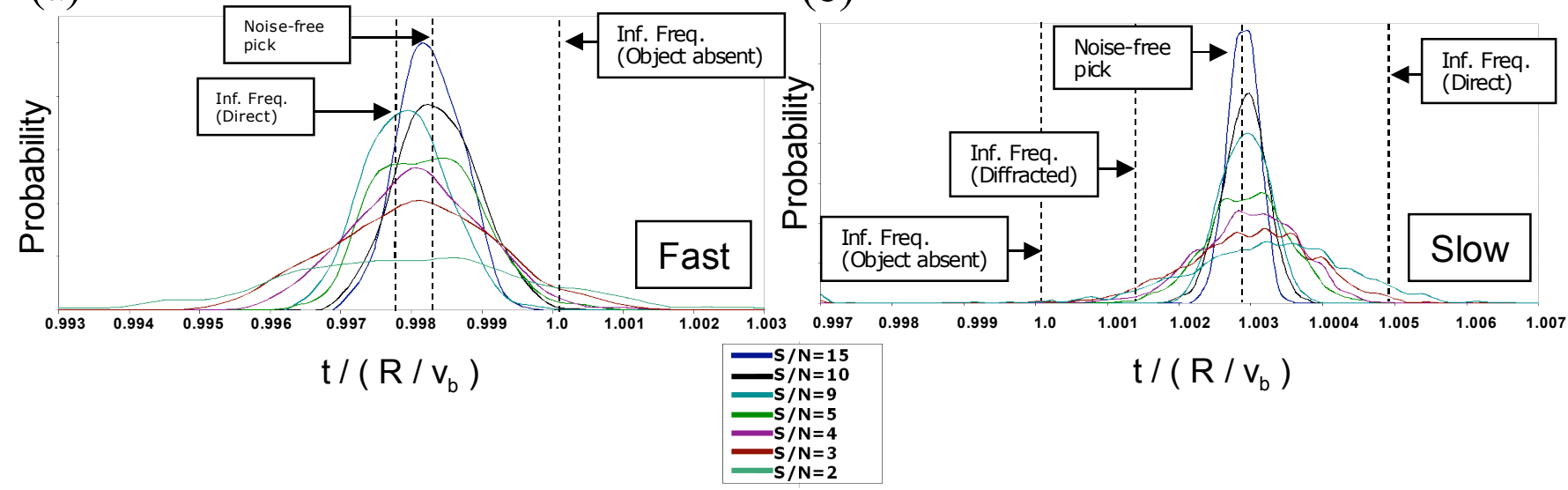

Figure 14. Histogram of 50\% threshold picking results for (a) fast and (b) slow object data at different $\mathrm{S} / \mathrm{N}$. The 0 degree trace was picked with many different noise realizations. The signal dominant frequency corresponds to a Fresnel zone approximately three times larger than the object. 


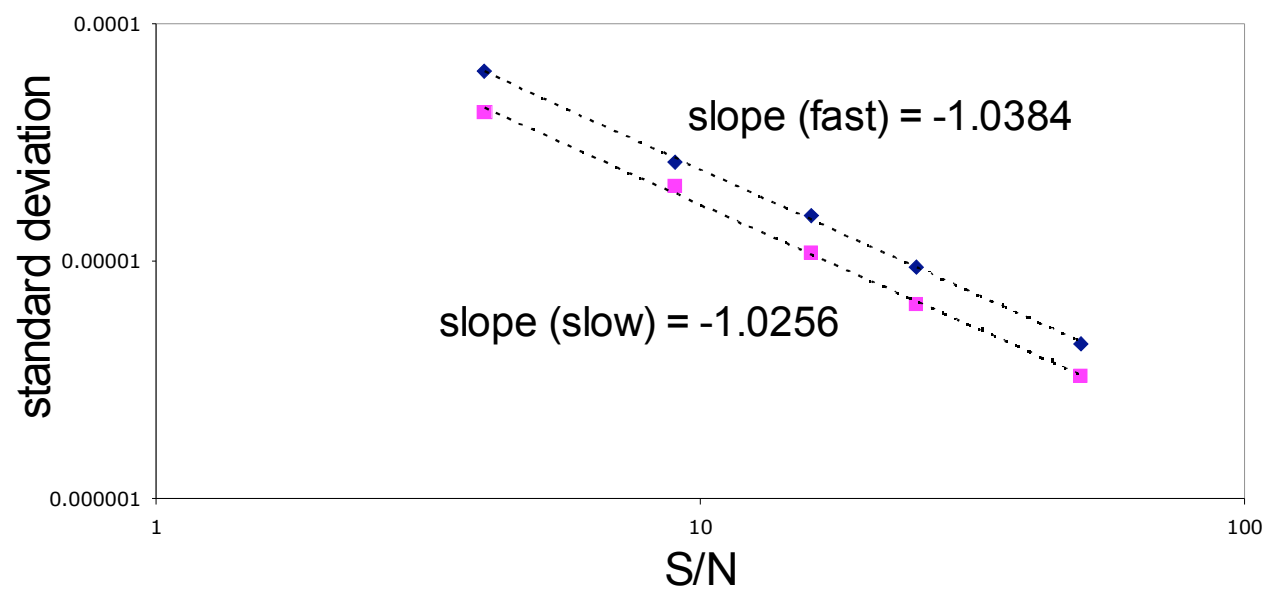

Figure 15. Standard deviation of pick distributions from Figure 14 as a function of S/N on logarithmic-logarithmic scale for fast (blue dots) and slow (pink dots) noisy picks. The best-fitting straight line (power law) is plotted and slope indicated. 
(a)

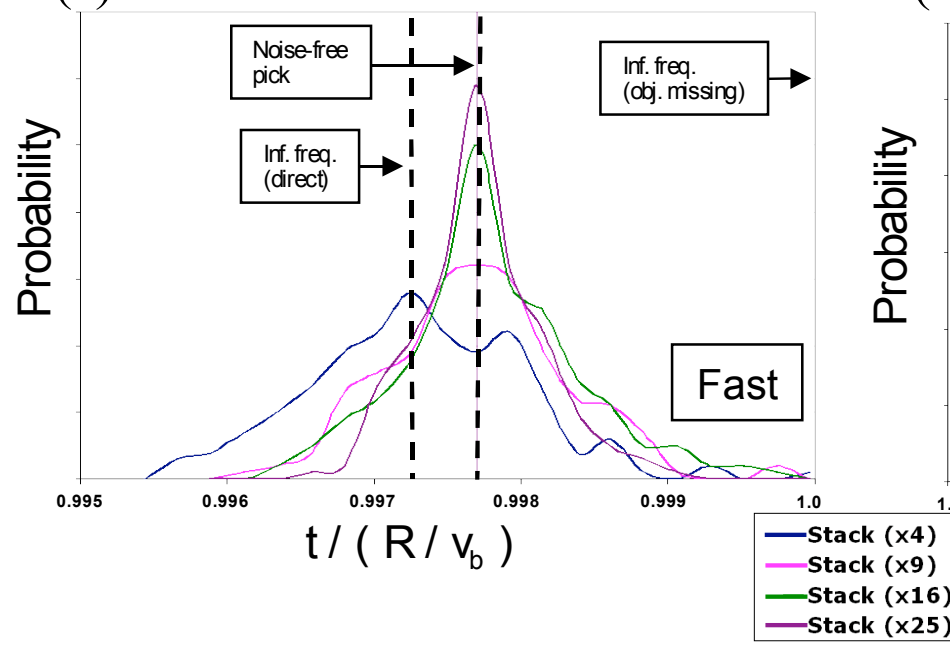

(b)

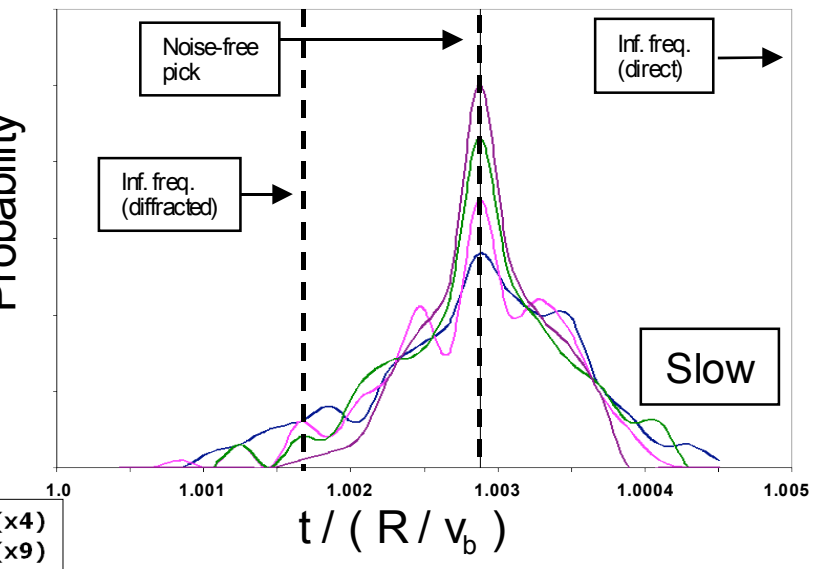

Figure 16. Histogram of 50\% threshold picking results for (a) fast and (b) slow object data at different stacking fold. Traces were originally generated with different noise at $\mathrm{S} / \mathrm{N}$ of 2 , multiple traces were stacked, and the stacked trace was picked. The signal dominant frequency corresponds to a Fresnel zone approximately three times larger than the object. 


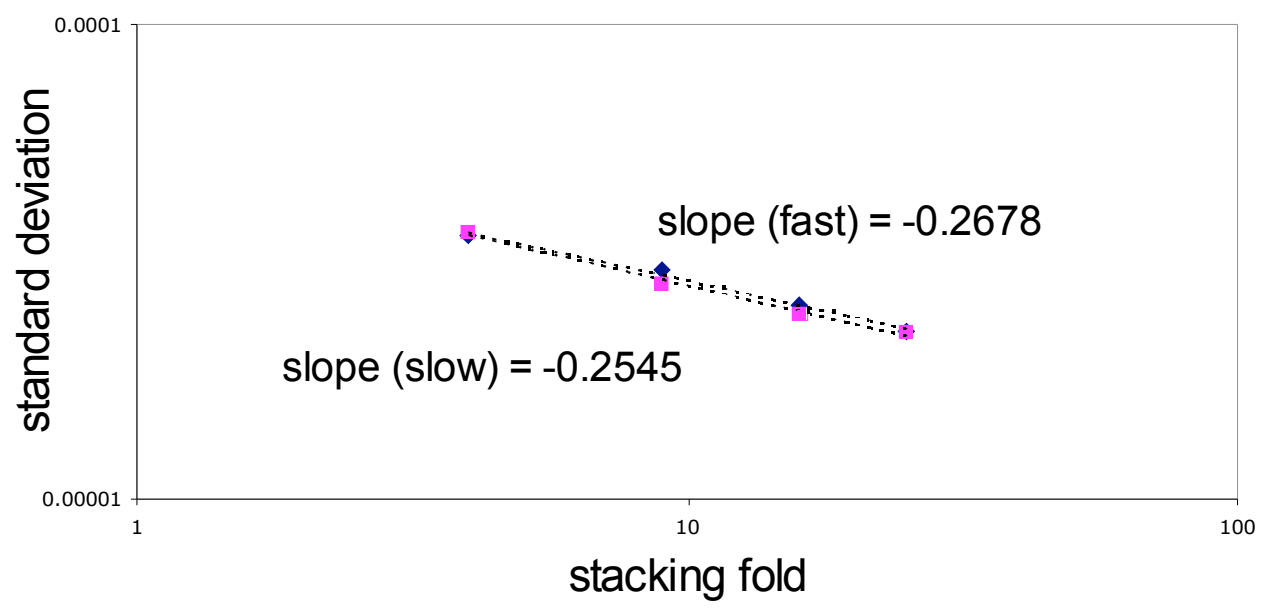

Figure 17. Standard deviation of pick distributions from Figure 16 as a function of stacking fold on logarithmic-logarithmic scale for fast (blue dots) and slow (pink dots) stacked picks. The best-fitting straight line (power law) is plotted and slope indicated. 
(a)

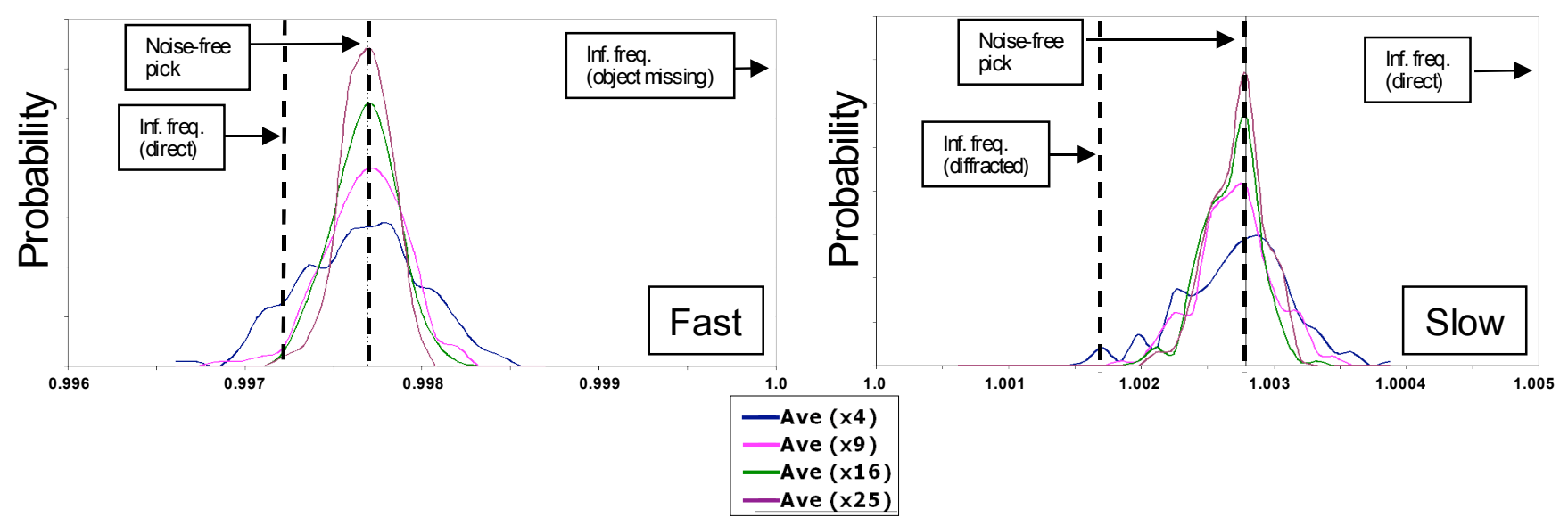

Figure 18 . Histogram of $50 \%$ threshold picking results for (a) fast and (b) slow object data at different averaging fold. Traces were originally generated with different noise at $\mathrm{S} / \mathrm{N}$ of 2 , the traces were picked, and the picks were averaged. The signal dominant frequency corresponds to a Fresnel zone approximately three times larger than the object. 


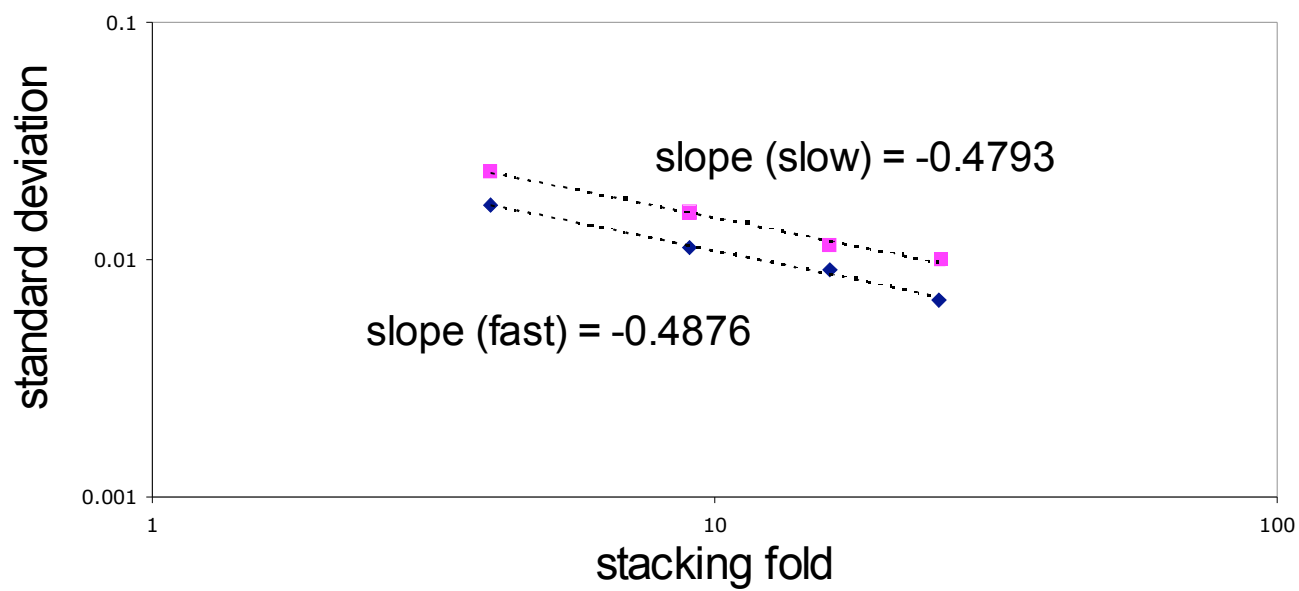

Figure 19. Standard deviation of pick distributions from Figure 18 as a function of averaging fold on logarithmic-logarithmic scale for fast (blue dots) and slow (pink dots) averaged picks. The best-fitting straight line (power law) is plotted and slope indicated. 

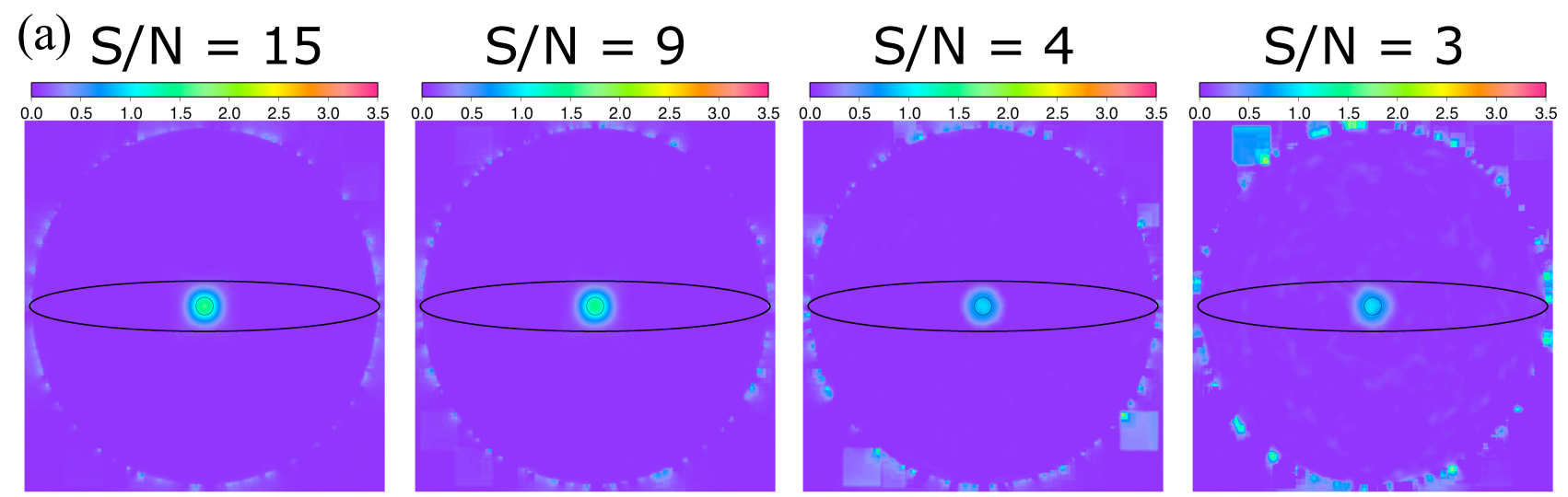

(b) $\mathrm{S} / \mathrm{N}=15$

$$
\mathrm{S} / \mathrm{N}=9
$$

$\mathrm{S} / \mathrm{N}=4$

$\mathrm{S} / \mathrm{N}=3$
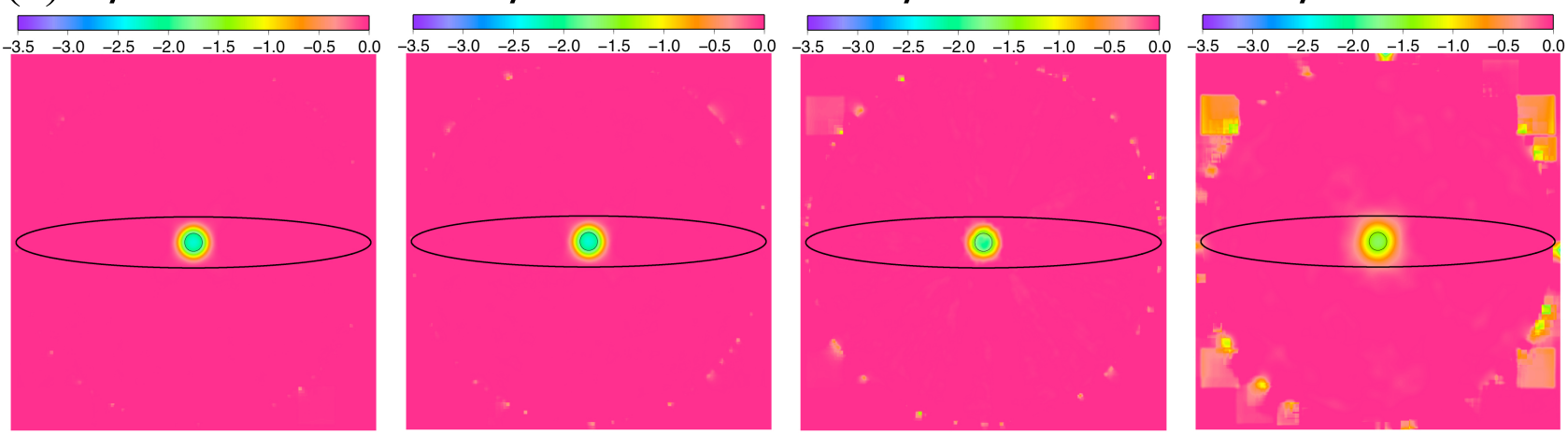

(c)

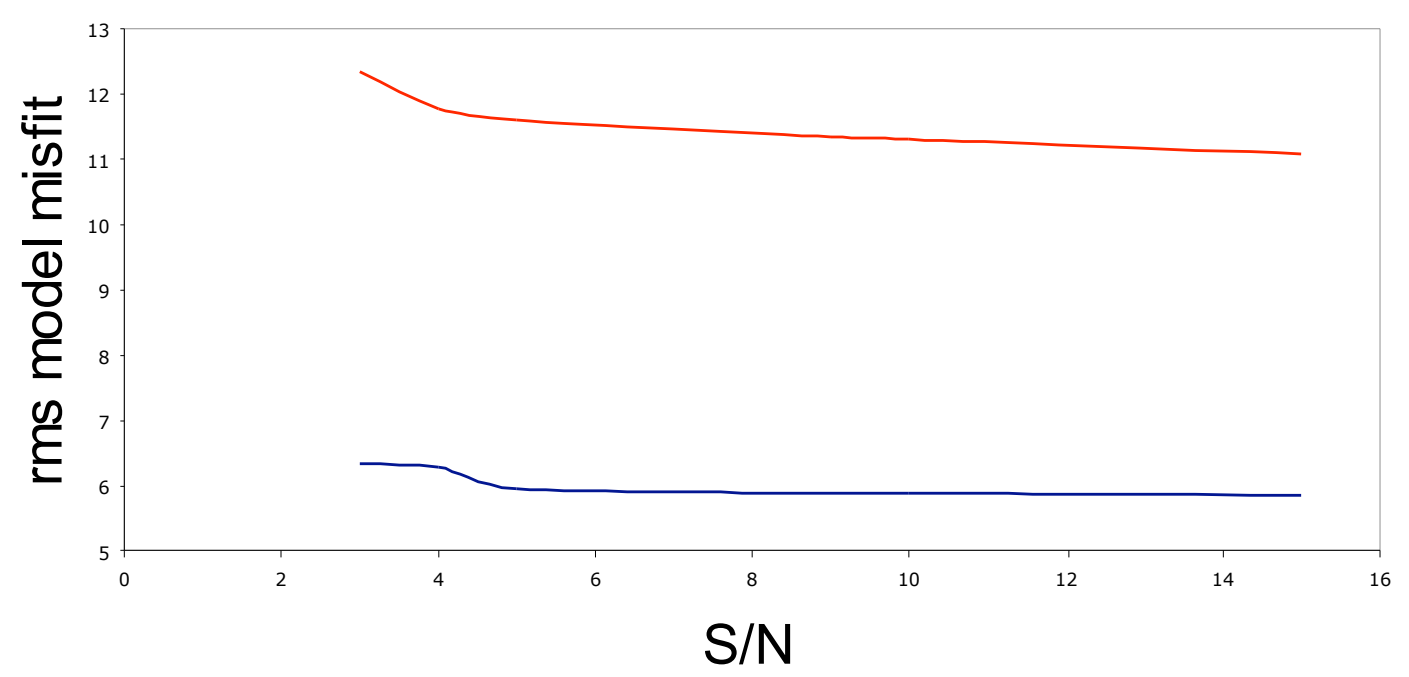

Figure 20. Example tomograms for (a) fast and (b) slow object noisy data at 360 sources and receivers. (c) Root-mean squared misfit from true model calculated for area within the source receiver and plotted as a function of $\mathrm{S} / \mathrm{N}$. Blue line is the misfit for fast object images and red line is the misfit for slow object images. 
(a) $S \& R=36$

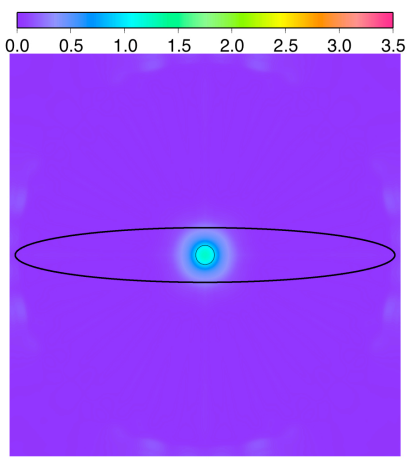

(b) $S \& R=36$

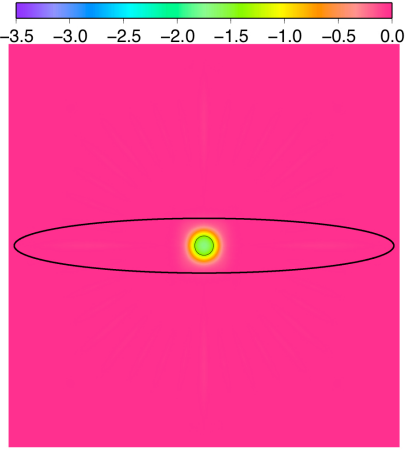

$S \& R=24$

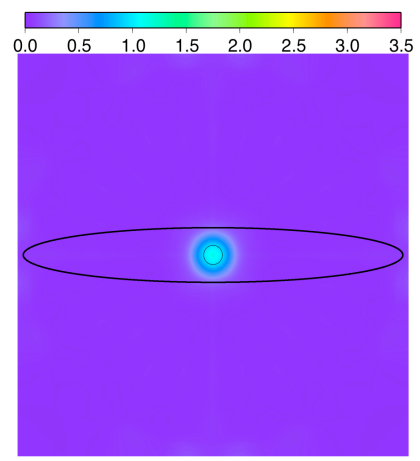

\section{$S \& R=24$}

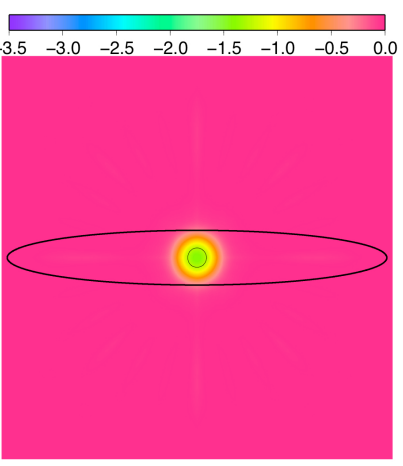

$S \& R=12$

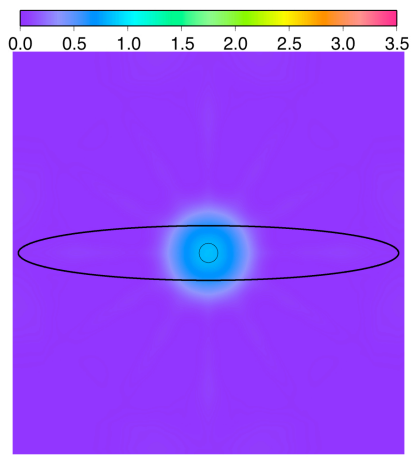

$S \& R=12$

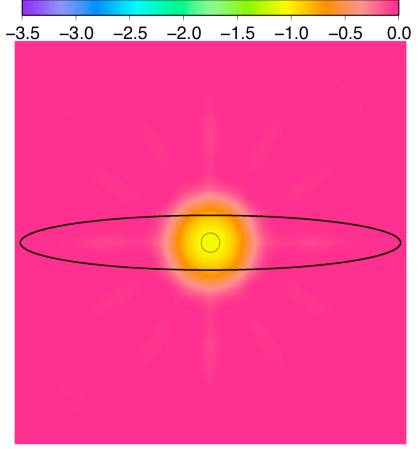

$S \& R=8$

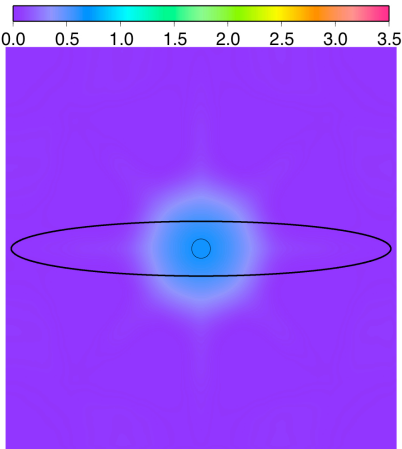

$S \& R=8$

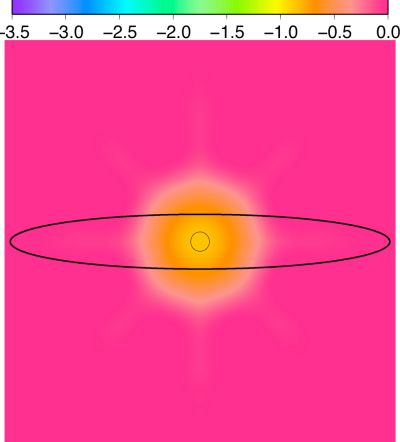

(c)

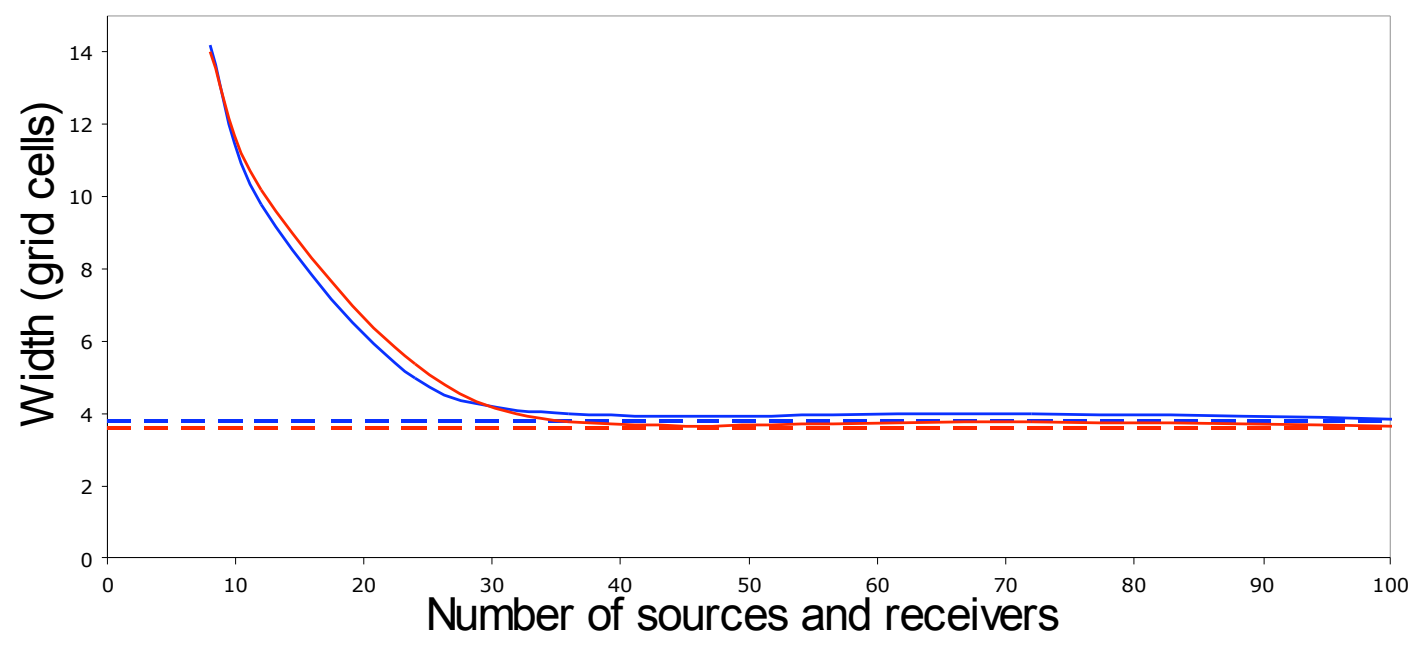

Figure 21. Example tomograms for noise-free (a) fast and (b) slow object 50\%

threshold picks with decreasing ray coverage. S\&R refers to the number of sources

and receivers. (c) Width of the object as a function of the number of sources and receiver; solid blue line is the measured width for the fast image and dashed blue is the width for 360 sources and receivers; solid red line is the measured width of the slow image and the dashed red is the width for 360 sources and receivers. Width is defined in the same manner as Figure 12. 


\section{$S \& R=360-S / N=3$}

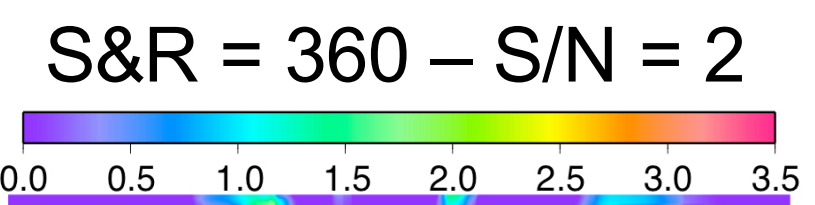

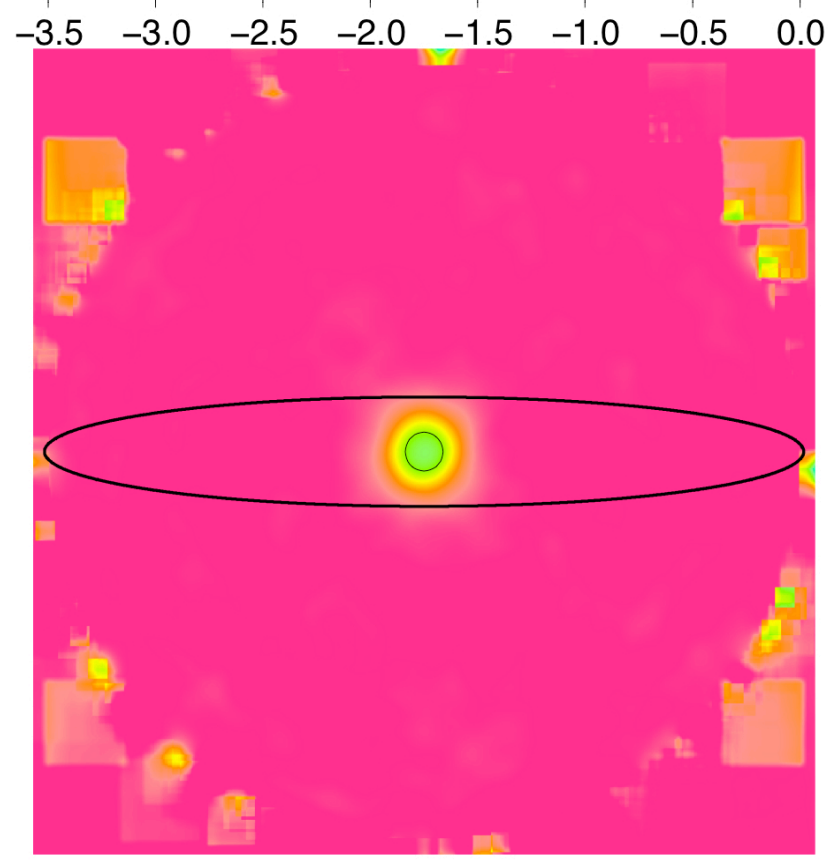

\section{Slow}

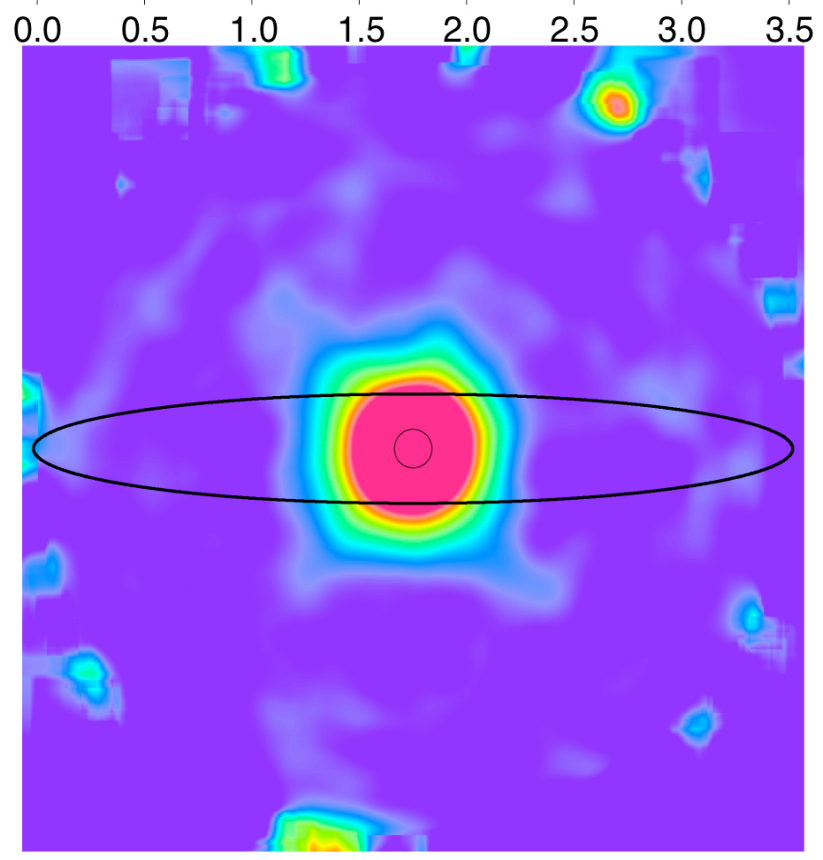

Fast

Figure 22. Example tomograms of fast and slow object data at low $\mathrm{S} / \mathrm{N}$ but with excellent ray coverage. (a) shows slow object data at $\mathrm{S} / \mathrm{N}$ of 3 and 360 sources and receivers. The object is very well imaged in terms of size, geometry, and position. (b) shows the fast object data at $\mathrm{S} / \mathrm{N}$ of 2 and 360 sources and receiver. The object is well imaged in position but poorly constrained in size and geometry. In both cases, individual traveltime are less accurate than the difference between absence and presence of the object, yet the object is successfully imaged. 
(a) $S \& R=72$

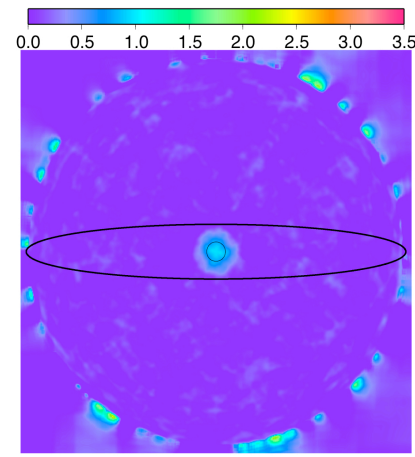

(b) $S \& R=72$

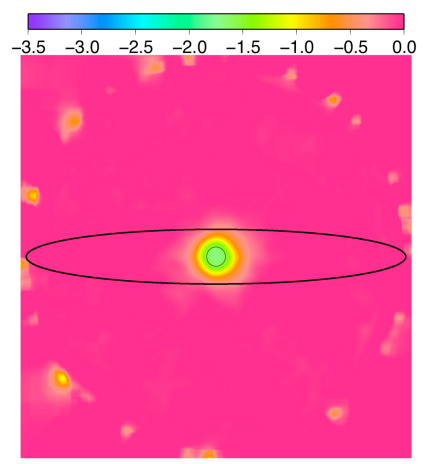

$S \& R=36$

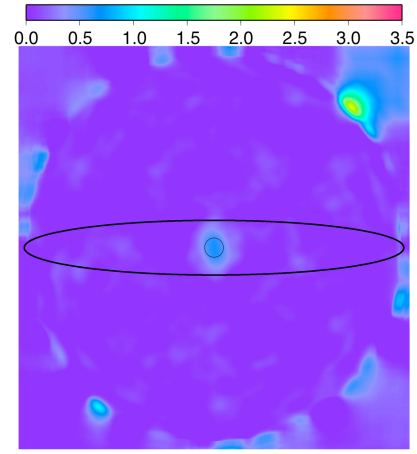

\section{$S \& R=36$}

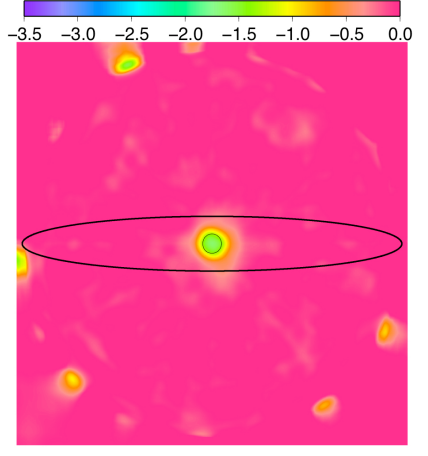

(c)

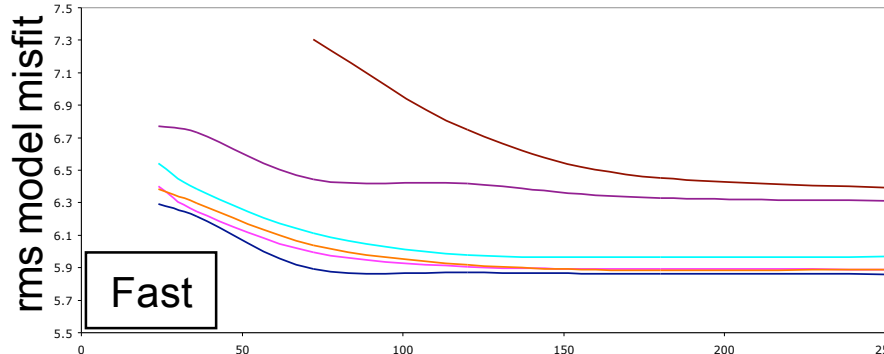

(d)

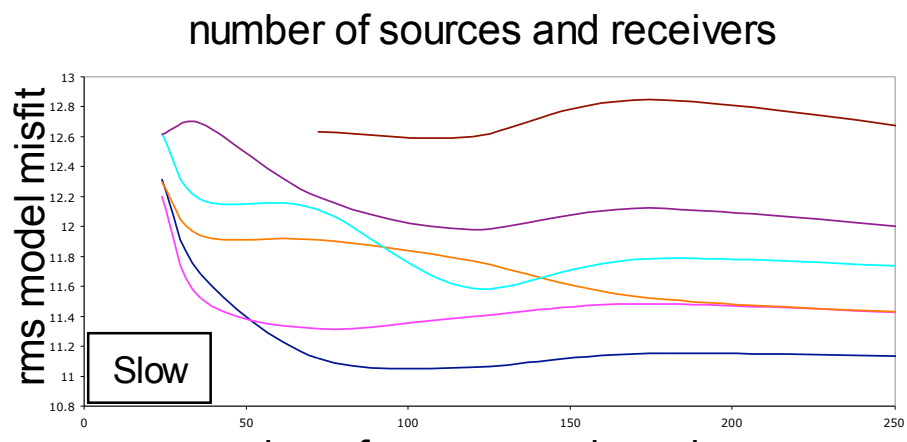

number of sources and receivers
$S \& R=24$

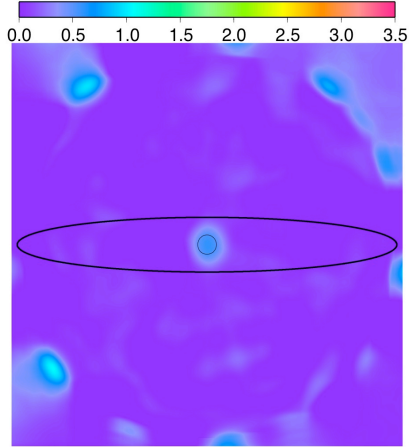

$S \& R=24$
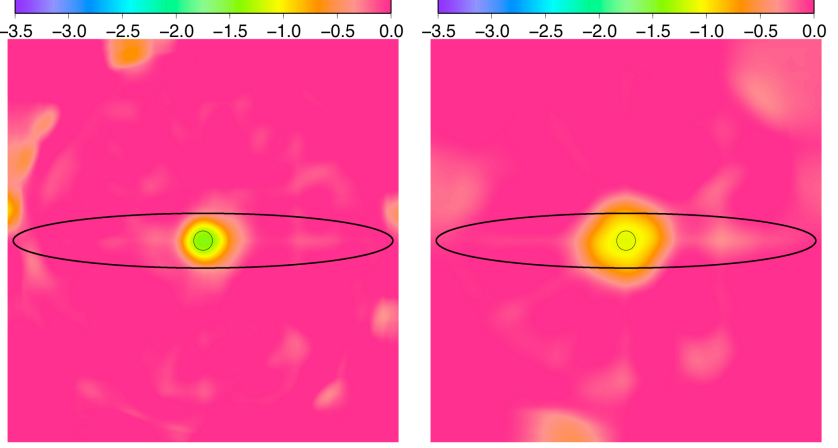

$S \& R=12$

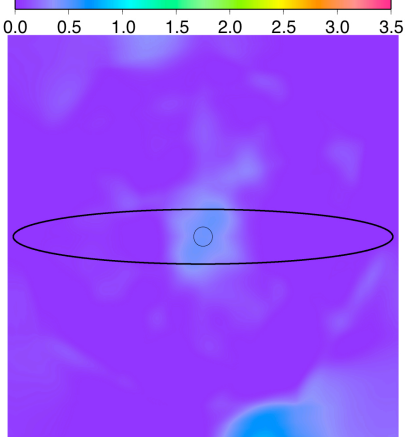

$S \& R=12$
$-\mathrm{S} / \mathrm{N}=\mathbf{1 5}$

$S / N=10$

$S / N=9$

$S / N=5$

$S / N=4$

$-\mathbf{S} / \mathbf{N}=\mathbf{3}$

Figure 23. Example tomograms for (a) fast and (b) slow object data at $\mathrm{S} / \mathrm{N}$ of 4 at different ray coverages. S\&R refers to the number of sources and receivers Root-mean squared misfit was calculated for the area inside the source-receiver circle for each source-receiver configuration at each $\mathrm{S} / \mathrm{N}$ relative to the true model. The rms model mis fit of each ray coverage configuration at a range of $\mathrm{S} / \mathrm{N}$ for the (c) fast and (d) slow object data. 